\title{
Quiver gauge theories and integrable lattice models
}

\author{
Junya Yagi \\ International School for Advanced Studies (SISSA), \\ via Bonomea 265, 34136 Trieste, Italy \\ INFN - Sezione di Trieste, \\ via Valerio 2, 34149 Trieste, Italy \\ E-mail: junya.yagi@sissa.it
}

ABSTRACT: We discuss connections between certain classes of supersymmetric quiver gauge theories and integrable lattice models from the point of view of topological quantum field theories (TQFTs). The relevant classes include $4 \mathrm{~d} \mathcal{N}=1$ theories known as brane box and brane tilling models, $3 \mathrm{~d} \mathcal{N}=2$ and $2 \mathrm{~d} \mathcal{N}=(2,2)$ theories obtained from them by compactification, and $2 \mathrm{~d} \mathcal{N}=(0,2)$ theories closely related to these theories. We argue that their supersymmetric indices carry structures of TQFTs equipped with line operators, and as a consequence, are equal to the partition functions of lattice models. The integrability of these models follows from the existence of extra dimension in the TQFTs, which emerges after the theories are embedded in M-theory. The Yang-Baxter equation expresses the invariance of supersymmetric indices under Seiberg duality and its lower-dimensional analogs.

KeYwords: Supersymmetric gauge theory, Brane Dynamics in Gauge Theories, Lattice Integrable Models, Topological Field Theories

ARXIV EPRINT: 1504.04055 


\section{Contents}

1 Introduction 1

2 Integrable lattice models from TQFTs with extra dimensions 5

2.1 Vertex models from TQFTs 5

$\begin{array}{lll}2.2 & \text { Integrability and extra dimensions } & 7\end{array}$

$\begin{array}{lll}2.3 & \text { IRF models } & 10\end{array}$

$\begin{array}{lll}2.4 & 3 \mathrm{~d} \text { TQFTs and the tetrahedron equation } & 11\end{array}$

$34 \mathrm{~d} \mathcal{N}=1$ quiver gauge theories $\quad 13$

$\begin{array}{lll}3.1 & \text { Brane box models } & 13\end{array}$

$\begin{array}{lll}3.2 & \text { R-symmetry and flavor symmetries } & 16\end{array}$

$\begin{array}{lll}3.3 & \text { Structure of a TQFT with extra dimension } & 19\end{array}$

3.4 Integrable lattice model for brane box models 22

4 Brane tiling models $\quad 26$

$53 \mathrm{~d} \mathcal{N}=2$ and $2 \mathrm{~d} \mathcal{N}=(2,2)$ quiver gauge theories 30

$5.13 \mathrm{~d}$ brane box models 31

5.2 2d brane box models 33

$62 \mathrm{~d} \mathcal{N}=(0,2)$ quiver gauge theories $\quad 36$

$6.1 \mathcal{N}=(0,2)$ theories related to brane box configurations 36

$6.2 \mathcal{N}=(0,2)$ theories related to brane tiling configurations $\quad 40$

6.3 Brane cube models 40

\section{Introduction}

The present work is motivated by an intriguing connection discovered in the past few years between supersymmetric quiver gauge theories and integrable lattice models in statistical mechanics.

In $[1,2]$, Bazhanov and Sergeev introduced an integrable spin model on a planar lattice, which generalizes many of previously known integrable lattice models such as the Kashiwara-Miwa [3] and chiral Potts [4-6] models. Soon after the first paper by Bazhanov and Sergeev appeared, Spiridonov [7] gave an interpretation of their model in terms of $\mathcal{N}=1$ quiver gauge theories in four dimensions. The relation to gauge theory was further elucidated by Yamazaki $[8,9]$, who realized that the relevant quiver gauge theories arise naturally from a particular class of brane configurations in string theory, called brane tilings [10]. From the gauge theory viewpoint, the lattice on which the spin model is defined is the quiver diagram, the partition function is the supersymmetric index, and the 
Yang-Baxter equation that guarantees the integrability of the model is a special instance of Seiberg duality.

This discovery, while quite remarkable, leaves us with a series of questions: Why are the supersymmetric indices of these theories captured by a lattice model? Why is this model integrable? Are there structures of integrable lattice models hidden in other theories?

In this paper we answer these questions, combining ideas from two equally stimulating connections uncovered in recent years. Of these connections, one lies between a certain class of quiver gauge theories and topological quantum field theories (TQFTs), whereas the other relates TQFTs and integrable lattice models. Our goal is to connect quiver gauge theories and integrable lattice models, and TQFTs provide a bridge between the two elements.

The first connection in question arises from the M5-brane construction of $4 \mathrm{~d} \mathcal{N}=2$ theories [11-13]. Consider a stack of M5-branes wrapped on $S^{1} \times S^{3} \times \Sigma$, where $\Sigma$ is a compact Riemann surface. In addition, we introduce M5-branes that intersect with these branes along submanifolds of the form $S^{1} \times S^{3} \times\left\{p_{i}\right\}$, with $p_{i}$ being points on $\Sigma$. If $\Sigma$ is small compared to the $S^{1}$ and $S^{3}$, this brane system is described at low energies by an $\mathcal{N}=2$ theory on $S^{1} \times S^{3}$. Often this is a quiver gauge theory. The path integral on the geometry $S^{1} \times S^{3}$ (after analytic continuation to Euclidean spacetime) computes the supersymmetric index of the theory. The most important property of this quantity is that it is invariant under continuous changes of the parameters of the theory. Since the geometry of $\Sigma$ encodes such parameters, it follows that the supersymmetric index is a topological invariant of $\Sigma$. In fact, it is equal to a correlation function $\left\langle\prod_{i} \mathcal{O}_{i}\left(p_{i}\right)\right\rangle$ in a TQFT on $\Sigma$, where $\mathcal{O}_{i}$ is a local operator representing the M5-brane inserted at $p_{i}$ [14]. If we consider a protected quantity different from the index on $S^{3}$ (such as the lens space index [15]), then we get a different TQFT.

The second connection refers to a general construction of integrable lattice models from TQFTs of a special kind, due to Costello [16, 17]. Given a 2d TQFT equipped with line operators, we can place it on a torus $T^{2}$ and wrap line operators around various 1-cycles that form a lattice. The topological invariance of the theory implies that the correlation function for this lattice of line operators coincides with the partition function of a statistical mechanics model defined on the same lattice. Under this correspondence, the Yang-Baxter equation translates to the statement that the correlator remains the same when a line operator is moved past the intersection of two other line operators. The structure of a TQFT itself is not strong enough to ensure this property since the move is not topologically trivial; the system may undergo a phase transition as the moved line hits and crosses the intersection point. If, however, the theory has "extra dimensions" along which the line can be shifted, the collision can be avoided and hence the YangBaxter equation holds. What is more, the correlator then carries continuous parameters, namely the positions of the line operators in the extra dimensions. In the context of integrable models, continuous parameters on which the Boltzmann weight depends are called spectral parameters, and their presence is essential for integrability. Thus, a $2 \mathrm{~d}$ TQFT with extra dimensions produces from line operators a solution of the Yang-Baxter equation with spectral parameter. 
We wish to understand the connection between brane tilings and integrable lattice models in light of these independent, though apparently related, developments in the relevant areas. To this end, it proves helpful to first study the case of the brane box construction [18], which is a precursor of the brane tiling construction and conceptually simpler.

The brane box construction is similar to the M5-brane construction described above. In this construction, we start with a stack of $N$ D5-branes on $S^{1} \times S^{3} \times T^{2}$, and put NS5branes on submanifolds of the form $S^{1} \times S^{3} \times C_{\alpha}$. Here $C_{\alpha}$ are 1-cycles of $T^{2}$, making up a lattice. At low energies, this brane configuration realizes a $4 \mathrm{~d} \mathcal{N}=1$ quiver gauge theory, which we refer to as a "brane box model," placed on $S^{1} \times S^{3}$. (Actually, $T^{2}$ can be replaced by any Riemann surface without breaking the $\mathcal{N}=1$ supersymmetry.) Just as we did for the M5-brane construction, we can relate this theory to a $2 \mathrm{~d}$ TQFT by considering its supersymmetric index. Adapted to the present situation, the argument used there shows that the supersymmetric index is given by a correlation function $\left\langle\prod_{\alpha} \mathcal{L}_{\alpha}\left(C_{\alpha}\right)\right\rangle$ in a TQFT on $T^{2}$, where $\mathcal{L}_{\alpha}\left(C_{\alpha}\right)$ is a line operator created by the NS5-brane wrapped around $C_{\alpha}$.

According to the construction of lattice models from TQFTs, this correlator coincides with the partition function of a lattice model. To establish the integrability of the model, we need extra dimensions along which line operators can move freely. An extra dimension indeed emerges as 11th dimension if we embed the brane system into M-theory by string dualities. Hence, we conclude that the supersymmetric index of a brane box model is equal to the partition function of an integrable lattice model. It turns out that the Yang-Baxter equation reduces to Seiberg duality for $\mathrm{SU}(N)$ SQCD with $2 N$ flavors.

Once the connection between the brane box construction and integrable lattice models is understood, the case of brane tilings is not so difficult. In this case we consider deformations of brane box configurations, in which we let the NS5-branes combine with the D5-branes over ribbon-shaped neighborhoods of the 1-cycles $C_{\alpha}$ in $T^{2}$. Such a deformed brane configuration still yields a $4 \mathrm{~d} \mathcal{N}=1$ quiver gauge theory, provided that the deformation meets a certain criterion. By the same reasoning as above, we deduce that the supersymmetric index of this theory is given by the correlation function of "thickened" line operators representing the ribbon neighborhoods, and equal to the partition function of an integrable lattice model. In this way the Bazhanov-Sergeev model arises from brane tilings. Again, the Yang-Baxter equation boils down to Seiberg duality, though this time the equation involves a sequence of four basic duality transformations.

So we have answers to the first two of our questions. The supersymmetric indices of theories constructed from brane tilings are captured by a lattice model since they are given by correlation functions of line operators in a TQFT, which in turn is a consequence of the nature of the brane construction and the fact that the index is a protected quantity. Furthermore, the integrability of this lattice model is guaranteed by the hidden extra dimension which emerges after the brane system is embedded in M-theory.

In sections $2-4$, we discuss in greater detail the connections summarized here among the brane box and brane tiling constructions, TQFTs with extra dimensions, and integrable lattice models. After reviewing in section 2 the construction of integrable lattice models from TQFTs with extra dimensions, we explain in section 3 how it can be applied to the supersymmetric indices of brane box models. We identify the associated integrable lattice 
model, and show that the Yang-Baxter equation for this model takes the form of Seiberg duality. In section 4 , we treat the case of brane tiling models.

Sections 5 and 6 are devoted to answering our third question, that is, finding more examples of quiver gauge theories whose supersymmetric indices are captured by integrable lattice models.

One way to produce more quiver gauge theories is to apply T-duality to the brane configurations discussed above. In this manner we get $3 \mathrm{~d} \mathcal{N}=2$ and $2 \mathrm{~d} \mathcal{N}=(2,2)$ theories. Being related to the $4 \mathrm{~d}$ parents by T-duality, the supersymmetric indices of these theories are also given by the partition functions of some integrable lattice models. In section 5, we will see that for these theories, the Yang-Baxter equation follows from lowerdimensional analogs of Seiberg duality, namely a variant of Aharony duality [19] in the $3 \mathrm{~d}$ case and Hori-Tong duality [20] in the $2 \mathrm{~d}$ case.

Perhaps more unexpected is that there are $2 \mathrm{~d} \mathcal{N}=(0,2)$ quiver gauge theories whose supersymmetric indices, or elliptic genera, exhibit integrability. They have half as many supercharges as the theories mentioned so far, and cannot be obtained by simple dimensional reduction from three or four dimensions.

In section 6 , we discuss three classes of such $\mathcal{N}=(0,2)$ theories. Two of them are $\mathcal{N}=(0,2)$ counterparts of the classes of $\mathcal{N}=(2,2)$ theories considered in section 5 . The Yang-Baxter equation for these classes identifies two theories related by Seiberg-like triality, discovered by Gadde, Gukov and Putrov [21]. The third class consists of $\mathcal{N}=(0,2)$ theories constructed from brane cube configurations [22], and actually gives rise to a $3 \mathrm{~d}$ lattice model. The integrability condition for this model is that its Boltzmann weight satisfies Zamolodchikov's tetrahedron equation [23, 24], which is the $3 \mathrm{~d}$ analog of the YangBaxter equation. Our analysis in this section will be somewhat incomplete, unfortunately. For the first two classes, we will demonstrate the integrability of the associated lattice models, but not identify the underlying brane constructions or TQFT structures whose existence is strongly suggested by the integrability. For the third class, on the other hand, we will describe the brane construction and associated lattice model, but not determine the corresponding solution of the tetrahedron equation. ${ }^{1}$

Having established the structures of integrable lattice models in several classes of quiver gauge theories, we should now ask what we can do with these structures. It is likely that knowledge accumulated in the area of integrable models provides new insights into these theories or quiver gauge theories in general. Conversely, tools from the gauge theory side, such as localization and $1 / N$ expansion, may help further elucidate the physics of integrable models.

Given the generality of the TQFT construction of integrable lattice models, we also expect that there are many more applications than those discussed in this paper. Below we describe just a few possibilities.

The NS5-branes in a brane box configuration can be mapped by dualities to either M5or M2-branes intersecting with a stack of M5-branes. These branes represent codimension-

\footnotetext{
${ }^{1} \mathrm{~A}$ different solution of the tetrahedron equation has been found recently by Gadde and Yamazaki [25]. Their solution is based on $\mathcal{N}=(0,2) \mathrm{SQCD}$ [21] with all flavor nodes having equal ranks. It remains to be seen whether this one may be understood from the perspective adopted in this paper.
} 
2 and -4 defects in $6 \mathrm{~d} \mathcal{N}=(2,0)$ superconformal field theory. From the $4 \mathrm{~d}$ point of view, they are domain walls and line operators in an $\mathcal{N}=2$ theory. ${ }^{2}$ This observation suggests that integrability plays a key role in the physics of defects in the $6 \mathrm{~d}$ theory and $4 \mathrm{~d} \mathcal{N}=2$ theories, and the ideas contained in this paper may be useful for understanding it. In this regard, we point out that the Yang-Baxter equation has made an unexpected appearance in studies of the moduli spaces of $\mathcal{N}=2$ theories compactified on $S^{1}[26,27]$.

Another important appearance of integrability is found in the Bethe/gauge correspondence [28-30] between $2 \mathrm{~d} \mathcal{N}=(2,2)$ theories and quantum integrable systems. It may be possible to connect that story to ours, by studying the supersymmetric vacua of $\mathcal{N}=(2,2)$ theories associated with brane box and brane tiling configurations.

Lastly, integrability features prominently in the AdS/CFT correspondence, and the constructions discussed in the present work may shed light on this aspect; after all, the developments of brane box and brane tiling techniques were motivated by the AdS/CFT correspondence. In fact, brane box models were studied at one-loop level in the planar limit in [31], and it was shown that at this level, their dilatation operator can be identified with the Hamiltonian of an integrable spin chain. This fact raises the hope that the integrability in the AdS/CFT correspondence may be understood in a framework that extends the one presented in this paper.

\section{Integrable lattice models from TQFTs with extra dimensions}

As explained in the introduction, the construction of integrable lattice models using line operators in TQFTs constitutes an essential ingredient for our argument. So let us begin by reviewing this construction. Our discussion mainly follows Costello's paper [16], to which we refer the reader for more details.

\subsection{Vertex models from TQFTs}

Consider a 2d TQFT equipped with a family of line operators parametrized by some continuous set, say $\mathbb{R}$ or $\mathbb{C}$. We denote by $\mathcal{L}_{r}(C)$ a line operator with parameter $r$, supported on an oriented closed curve $C$. Let us place this TQFT on a torus $T^{2}$, and choose 1-cycles $A_{1}, \ldots, A_{m}, B_{1}, \ldots, B_{n}$ that form an $m \times n$ lattice on $T^{2}$. The case with $(m, n)=(2,3)$ is illustrated in figure 1 . We are interested in the correlation function

$$
Z\left(\left\{r_{\alpha}\right\},\left\{s_{\beta}\right\}\right)=\left\langle\prod_{\alpha=1}^{m} \mathcal{L}_{r_{\alpha}}\left(A_{\alpha}\right) \prod_{\beta=1}^{n} \mathcal{L}_{s_{\beta}}\left(B_{\beta}\right)\right\rangle
$$

of line operators wrapped around these cycles.

We compute this correlation function by breaking up the torus into smaller rectangular pieces, as shown with dotted lines in figure 1. The idea is that we first perform the path integral on each of these pieces, and then glue the results together to reconstruct the path integral on $T^{2}$.

\footnotetext{
${ }^{2}$ We can obtain any $\mathcal{N}=2$ theory of class $\mathcal{S}[12,13]$ from the brane box configuration (3.1) by replacing $T^{2}$ with the relevant Riemann surface and introducing D5-branes in the 012357 directions.
} 


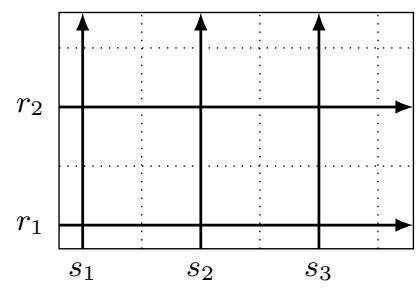

Figure 1. A $2 \times 3$ lattice of line operators on a torus. The dotted lines divide the lattice into rectangular pieces, each of which contains two intersecting segments of line operators.

Each piece in this decomposition is, topologically, a square with two line operators crossing in the middle:

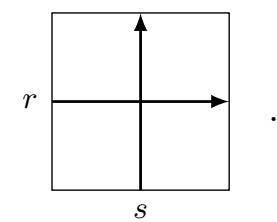

Using the topological invariance of the theory, we can deform it as

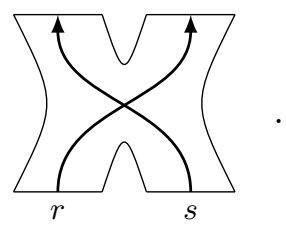

Let $V_{r}$ be the space of states on a finite interval intersected by $\mathcal{L}_{r}$. Intuitively, $V_{r}$ is the Hilbert space of an open string with a particle attached whose worldline is the line operator $\mathcal{L}_{r}$. In this language, the deformed picture shows an initial state of two open strings in $V_{r} \otimes V_{s}$ evolving into a final state in $V_{s} \otimes V_{r}$ that results from interaction in the middle. The original picture may be regarded as a particular case where the curved boundaries are shrunk to points. Thus, the path integral on the square produces a linear map

$$
\check{R}(r, s): V_{r} \otimes V_{s} \longrightarrow V_{s} \otimes V_{r} .
$$

We call $\check{R}$ the $R$-matrix of the TQFT with line operators.

Choosing a basis $\left\{e_{r, i}\right\}$ for $V_{r}$ for all $r$, we can represent $\check{R}$ by its matrix elements. The matrix element $\check{R}_{i_{1} j_{1}}^{j_{2} i_{2}}(r, s)$ is the scattering amplitude for a process in which the initial state $e_{r, i_{1}} \otimes e_{s, j_{1}}$ ends up in the final state $e_{s, j_{2}} \otimes e_{r, i_{2}}$. We represent it pictorially as

$$
\check{R}_{i_{1} j_{1}}^{j_{2} i_{2}}(r, s)=r \underset{s}{j_{2} \uparrow_{i_{1}} i_{2}} \longrightarrow .
$$


Then, the correlation function (2.1) is given by the formula

$$
Z\left(\left\{r_{\alpha}\right\},\left\{s_{\beta}\right\}\right)=\sum_{\left\{i_{\alpha, \beta}\right\}} \sum_{\left\{j_{\beta, \alpha}\right\}} \prod_{\alpha=1}^{m} \prod_{\beta=1}^{n} r_{\alpha} \stackrel{j_{\beta, \alpha+1}}{\stackrel{j_{\alpha, \beta}}{i_{j_{\alpha, \beta+1}}}}
$$

with the periodic boundary conditions $i_{\alpha, n+1}=i_{\alpha, 1}$ and $j_{\beta, m+1}=j_{\beta, 1}$. The right-hand side is what we get by gluing a collection of pieces like the picture (2.2) to reconstruct the line operators wrapped on the torus.

By representing the R-matrix as above, we are treating the open strings as if their physical degrees of freedom are carried solely by the particles attached to them, or equivalently, by the edges of the lattice. In words, the formula (2.6) instructs us to do the following. First, choose a state on every edge of the lattice. Next, compute the probability amplitude for this configuration of states by multiplying the corresponding R-matrix elements. Finally, sum over all possible such configurations to find the answer.

This procedure is, in fact, precisely how the partition function of a vertex model in statistical mechanics is defined. In a vertex model, state variables are assigned to the edges of a lattice, and the interaction takes place at the vertices. The total energy $E$ of the system is the sum of interaction energies at the vertices, so the Boltzmann weight $e^{-\beta E}$ factorizes into weight factors associated to the vertices. These factors are matrix elements of $\check{R}$. In this context, $r$ is called the spectral parameter of the model.

In conclusion, we have found that the correlation function (2.1) for a lattice of line operators in a $2 \mathrm{~d}$ TQFT coincides with the partition function of a vertex model defined on the same lattice, with the line operator parameter playing the role of a spectral parameter.

\subsection{Integrability and extra dimensions}

Let us fix the parameters $\left\{s_{\beta}\right\}$ in the lattice of line operators under consideration. Viewing the vertical direction of the torus as the time direction, we define the Hilbert space of the vertex model to be the tensor product ${ }^{3}$

$$
\mathcal{H}=\bigotimes_{\beta=1}^{n} V_{s_{\beta}}
$$

Furthermore, we introduce the row-to-row transfer matrix

$$
T(r)=\operatorname{Tr}_{V_{r}}\left(\check{R}\left(r, s_{1}\right){ }^{\circ} V_{r} \cdots{ }^{\circ} V_{r} \check{R}\left(r, s_{n}\right)\right) .
$$

\footnotetext{
${ }^{3}$ This is the space of states on the disjoint union of $n$ intervals, with $\beta$ th interval intersected by $\mathcal{L}_{s_{\beta}}$. The actual Hilbert space of the TQFT is generally smaller than $\mathcal{H}$, since the degrees of freedom associated with the endpoints ("Chan-Paton factors") must match between adjacent intervals.
} 
It is an endomorphism of $\mathcal{H}$, with matrix elements

$$
T_{j_{1,1}, \ldots, j_{n, 1}}^{j_{1,2}, \ldots, j_{n, 2}}(r)=\sum_{\left\{i_{\beta}\right\}} \prod_{\beta=1}^{n} r \stackrel{j_{\beta, 2}}{\uparrow_{i_{\beta}} i_{s_{\beta}} i_{\beta+1}}
$$

In terms of the transfer matrix, the partition function (2.6) is written as

$$
Z\left(\left\{r_{\alpha}\right\},\left\{s_{\beta}\right\}\right)=\operatorname{Tr}_{\mathcal{H}} \prod_{\alpha=1}^{m} T\left(r_{\alpha}\right)=\sum_{\left\{j_{\beta, \alpha}\right\}} \prod_{\alpha=1}^{m} T_{j_{1, \alpha}, \ldots, j_{n, \alpha}}^{j_{1, \alpha+1}, \ldots, j_{n, \alpha+1}}\left(r_{\alpha}\right)
$$

In a TQFT, time evolution is trivial unless the state hits some operator at some point in time. We may think of $T(r)$ as the time evolution operator induced by $\mathcal{L}_{r}$.

We say that the vertex model on the lattice of line operators is integrable if $T(r)$ is analytic in $r$ and

$$
\left[T(r), T\left(r^{\prime}\right)\right]=0
$$

for all $r, r^{\prime}$. The rationale for this terminology is that by expanding $T(r)$ in powers of $r$, we get a tower of operators that commute with one another and with the time evolution operator.

A sufficient condition for integrability is that the R-matrix has the following two properties. The first is that $\check{R}(r, s)$ is an isomorphism for $r \neq s$, with the inverse being $\check{R}(s, r)$ :

$$
\check{R}(r, s) \check{R}(s, r)=1 \text {. }
$$

The second is that $\check{R}$ satisfies the Yang-Baxter equation:

$$
\check{R}(s, t) \check{R}(r, t) \check{R}(r, s)=\check{R}(r, s) \check{R}(r, t) \check{R}(s, t) .
$$

Each factor on the two sides of the equation is regarded as an operator on a tensor product of $V_{r}, V_{s}$ and $V_{t}$. For example, $\check{R}(r, t)$ on the left-hand side is a map from $V_{s} \otimes V_{r} \otimes V_{t}$ to $V_{s} \otimes V_{t} \otimes V_{r}$.

In the TQFT language, the identity (2.12) means that two tangled line operators can be straightened out:

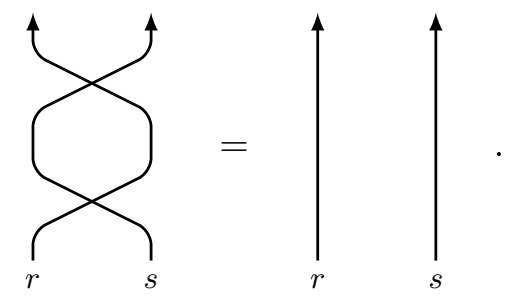


The Yang-Baxter equation, on the other hand, expresses invariance under moving a line operator past the intersection of two other line operators:

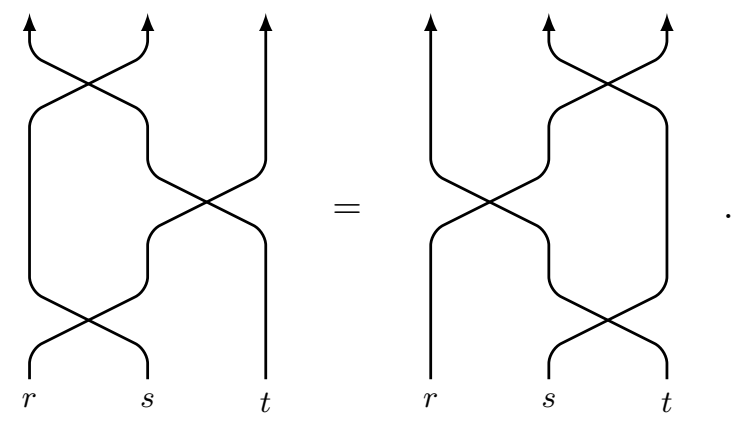

To see that these properties imply the commutativity of transfer matrices, consider the following move of line operators:

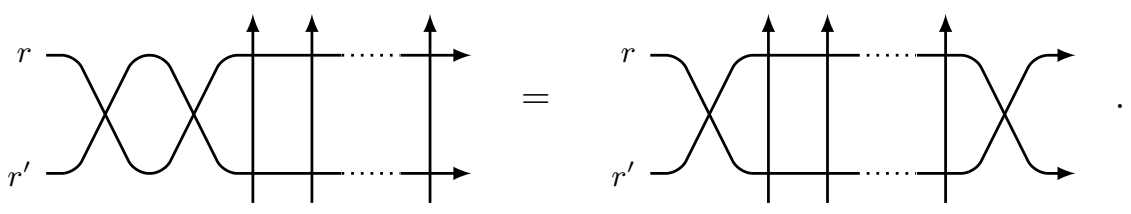

Taking the trace over $V_{r} \otimes V_{r^{\prime}}$ of both sides, and using the identity (2.14) and the cyclic property of the trace, we find that the left- and right-hand sides become $T(r) T\left(r^{\prime}\right)$ and $T\left(r^{\prime}\right) T(r)$, respectively.

In general, a 2d TQFT does not have the above properties. Physics may change discontinuously under the relevant moves due to phase transitions, as the topology of the line operators does not remain the same. Imagine, however, that there are "extra dimensions" hidden in the above pictures. For instance, the direction perpendicular to the page may provide one. The line operators can then sit at different points in the extra dimensions. If so, these moves are topologically trivial and hence the R-matrix satisfies the desired properties. An argument of this sort is familiar from studies of knot invariants, which may be approached either from the perspective of 3d TQFTs or lattice models [32, 33].

The above observation motivates us to introduce the notion of extra dimensions to TQFTs. Consider a $D$-dimensional quantum field theory formulated on $\Sigma \times M$, where $\Sigma$ is any $d$-manifold and $M$ is a fixed $(D-d)$-manifold. We say that the theory is a d-dimensional TQFT with extra dimensions if it is topological on $\Sigma{ }^{4}$ We refer to $M$ as the internal space of the theory. This definition is very much in the same spirit as the notion of a $2 \mathrm{~d}$ conformal field theory "valued in $4 \mathrm{~d}$ quantum field theories" [47] developed in connection to the $6 \mathrm{~d}$ construction of $4 \mathrm{~d} \mathcal{N}=2$ theories of class $\mathcal{S}$ [12, 13]. In that case,

\footnotetext{
${ }^{4}$ Although such theories might sound exotic, actually many have already been studied before. Typically, they are constructed from supersymmetric theories by topological twisting along $\Sigma$. $2 \mathrm{~d}$ examples are Costello's theory mentioned below, $4 \mathrm{~d} \mathcal{N}=2$ superconformal gauge theories with $M$ being any Riemann surface [34], 5d $\mathcal{N}=2$ super Yang-Mills theory with $M=S^{3}$ [35], and $6 \mathrm{~d} \mathcal{N}=(2,0)$ superconformal field theory with $M=S^{1} \times S^{3}$ [14]. 3d examples include $5 \mathrm{~d} \mathcal{N}=2$ super Yang-Mills theory with $M=\mathbb{R}_{\epsilon}^{2}[36,37]$ and $S^{2}$ [38-40], $6 \mathrm{~d} \mathcal{N}=(2,0)$ theory with $M=S^{1} \times S^{2}$ [41], $S^{3}$ [42], and more general lens spaces $L(p, q)$ [43]. $4 \mathrm{~d}$ examples are $6 \mathrm{~d} \mathcal{N}=(2,0)$ theory with $M$ any Riemann surface [44-46].
} 
however, the choice of the $4 \mathrm{~d}$ spacetime is not part of the definition. By contrast, here we demand the theory to be topologically invariant on $\Sigma$ for a specific choice of $M$.

Now we come to the main point of our discussion. Suppose that a $2 \mathrm{~d}$ TQFT with extra dimensions has line operators. Place the theory on $T^{2}$ and wrap line operators around 1cycles $A_{\alpha}$ and $B_{\beta}$. In the internal space $M$, the 1-cycles are located at some points $x_{\alpha}$ and $y_{\beta}$, respectively. The situation is similar to what we considered at the beginning of this section, but unlike that case, this time we do not assume that the line operators carry a continuous parameter. To highlight the difference, let us consider the extreme case that the theory has only one type of line operator, $\mathcal{L}$. Repeating the same argument, we arrive at the conclusion that the correlation function

$$
Z\left(\left\{x_{\alpha}\right\},\left\{y_{\beta}\right\}\right)=\left\langle\prod_{\alpha=1}^{m} \mathcal{L}\left(A_{\alpha} \times\left\{x_{\alpha}\right\}\right) \prod_{\beta=1}^{n} \mathcal{L}\left(B_{\beta} \times\left\{y_{\beta}\right\}\right)\right\rangle
$$

is given by the partition function of a vertex model defined on the lattice of line operators. As we have just seen, the presence of extra dimensions guarantees that the R-matrix is invertible and satisfies the Yang-Baxter equation.

A beautiful insight of Costello [16, 17] is that even though the line operator lacks a continuous parameter, the R-matrix still depends on such parameters: the positions $x_{\alpha}, y_{\beta}$ of line operators in $M$. Thus, the vertex model is integrable if the transfer matrix varies analytically on $M$. Based on this idea, it was shown in [17] that a special case of the 6 -vertex model corresponding to the XXX spin chain, and its generalizations based on Lie algebras other than $\mathfrak{s l}_{2}$, arise from a $2 \mathrm{~d}$ TQFT with extra dimensions whose internal space $M=\mathbb{C P}^{1}$. This TQFT is obtained from a deformed and topologically twisted version of $4 \mathrm{~d}$ $\mathcal{N}=1$ super Yang-Mills theory, and the line operators used in the construction are Wilson lines. The extra dimensions therefore elegantly explain not only why the Yang-Baxter equation holds for these models, but also where the spectral parameter comes from.

Speaking of extra dimensions, it should be noted that although only line operators were considered in our discussion, we could as well use higher-dimensional operators that have codimension at least two in $\Sigma \times M$. This is because after we wrap them on 1-cycles on $\Sigma$, there is still room in $M$ for them to avoid one another. In our main examples, we will make use of codimension- 2 defects in a $6 \mathrm{~d}$ theory.

\section{$2.3 \quad$ IRF models}

In the construction described above, we obtained a vertex model from line operators by letting the midpoints of open strings represent all physical degrees of freedom. In many cases, however, degrees of freedom really reside on (and only on) the endpoints. In such cases it is more efficient to reformulate the vertex model as an interaction-round-a-face (IRF) model.

In an IRF model, state variables live on the vertices of a lattice, and interaction takes place among vertices connected by edges that surround a face. We use letters $a, b, \ldots$ to denote state variables. For a square lattice, the Boltzmann weight for the interaction is 
denoted as

$$
W\left(\begin{array}{ll}
d & c \\
a & b
\end{array} \mid r, s\right)=r \text { (d) }
$$

The dashed oriented lines are rapidity lines, and make up the dual lattice. The parameters $r, s$ are spectral parameters, also called rapidities. An IRF model is a vertex model on the dual lattice, in which the state space assigned to an edge is the direct product of two spaces and many elements of the R-matrix vanish.

From the TQFT point of view, rapidity lines are line operators, and the rapidities are their parameters. The Yang-Baxter equation (2.15) for an IRF model is

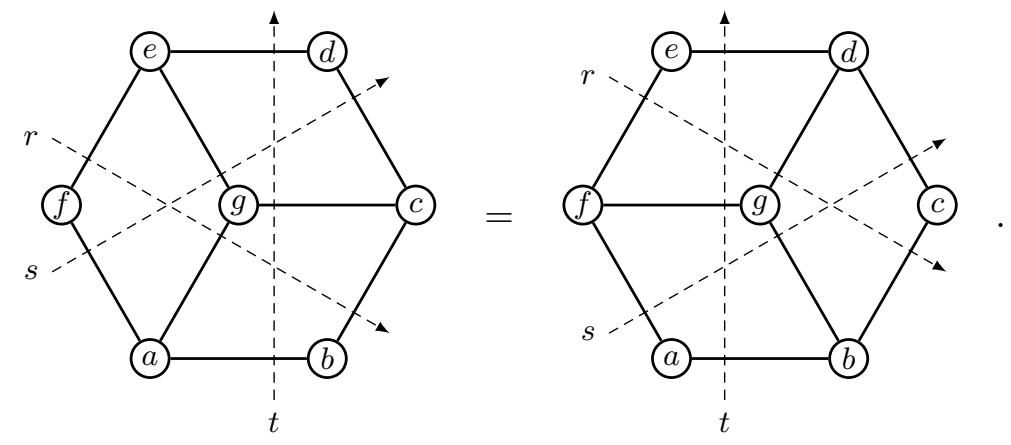

In terms of the Boltzmann weight, the equation reads

$$
\begin{aligned}
\sum_{g} W\left(\begin{array}{ll}
e & d \\
g & c
\end{array} \mid s, t\right) W\left(\begin{array}{ll}
g & c \\
a & b
\end{array} \mid\right. & r, t) W\left(\begin{array}{ll}
e & g \\
f & a
\end{array} \mid r, s\right) \\
& =\sum_{g} W\left(\begin{array}{ll}
d & c \\
g & b
\end{array} \mid r, s\right) W\left(\begin{array}{ll}
e & d \\
f & g
\end{array} \mid r, t\right) W\left(\begin{array}{ll}
f & g \\
a & b
\end{array} \mid s, t\right) .
\end{aligned}
$$

\section{$2.43 d$ TQFTs and the tetrahedron equation}

As a generalization, we can construct higher-dimensional lattice models using codimension1 defects in a $d$-dimensional TQFT with $d>2$. Placing the theory on a $d$-torus $T^{d}$ and putting these defects on $(d-1)$-cycles that form a $d$-dimensional lattice, we get a vertex model on this lattice. If the theory has extra dimensions, the R-matrix of the model satisfies a $d$-dimensional analog of the Yang-Baxter equation.

To be concrete, let us take $d=3$. In this case, codimension- 1 defects are surface operators, and three of them intersect at a vertex of the lattice. A cubic neighborhood of a vertex looks like

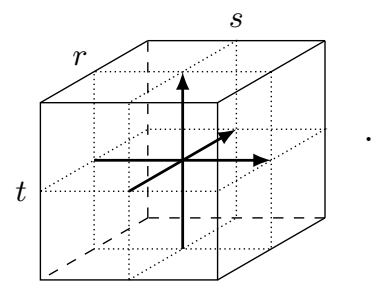


The orientations of the lattice edges specify those of the surface operators (drawn with dotted lines in the picture). Viewing this picture as the worldvolumes of three faces of the cube traveling in a diagonal direction, we see that the R-matrix $R(r, s, t)$ produced by the path integral on this cube is an endomorphism of $V_{r, s} \otimes V_{s, t} \otimes V_{t, r}$, where $V_{r, s}$ is the Hilbert spaces on a square intersected by two surface operators with parameters $r$ and $s$. Pictorially, the R-matrix is represented as

$$
R(r, s, t)=\underset{(s, t)}{\longrightarrow}
$$

To avoid clutter, in what follows we will suppress spectral parameters in this kind of pictures.

The 3d analog of the Yang-Baxter equation is Zamolodchikov's tetrahedron equation $[23,24]$, obtained by moving one of four surface operators that form a tetrahedron:

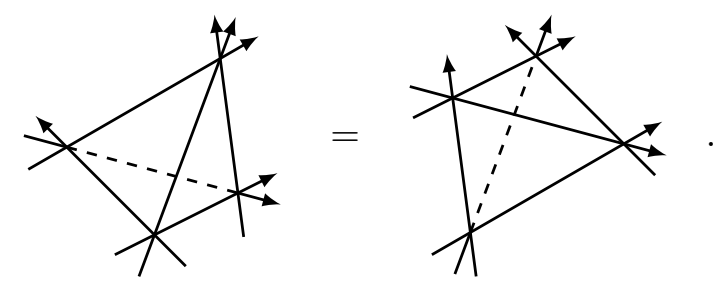

The tetrahedron equation implies the commutativity of layer-to-layer transfer matrices at different values of the spectral parameter.

When the state variables may be considered as living on the cubes surrounded by surface operators, a vertex model can be reformulated as an interaction-round-a-cube (IRC) model [48]. The Boltzmann weight of an IRC model is denoted as

$$
W(a|e, f, g| b, c, d \mid h)=\overbrace{\text { (b) }}^{(b)} \text { (d) }
$$

The tetrahedron equation for an IRC model is

$$
\begin{aligned}
& \begin{array}{c}
\sum_{d} W\left(a_{4}\left|c_{1}, c_{3}, c_{2}\right| b_{3}, b_{2}, b_{1} \mid d\right) W\left(c_{1}\left|a_{3}, b_{1}, b_{2}\right| d, c_{6}, c_{4} \mid b_{4}\right) \\
\times W\left(b_{1}\left|c_{4}, c_{3}, d\right| b_{3}, b_{4}, a_{2} \mid c_{5}\right) W\left(d\left|b_{4}, b_{3}, b_{2}\right| c_{2}, c_{6}, c_{5} \mid a_{1}\right) \\
=\sum_{d} W\left(b_{1}\left|c_{4}, c_{3}, c_{1}\right| a_{4}, a_{3}, a_{2} \mid d\right) W\left(c_{1}\left|a_{3}, a_{4}, b_{2}\right| c_{2}, c_{6}, d \mid a_{1}\right) \\
\times W\left(a_{4}\left|d, c_{3}, c_{2}\right| b_{3}, a_{1}, a_{2} \mid c_{5}\right) W\left(d\left|a_{3}, a_{2}, a_{1}\right| c_{5}, c_{6}, c_{4} \mid b_{4}\right) .
\end{array} \\
& \times
\end{aligned}
$$

Graphically, it takes the form of equivalence between two ways of dividing a dodecahedron 
into four hexahedra:

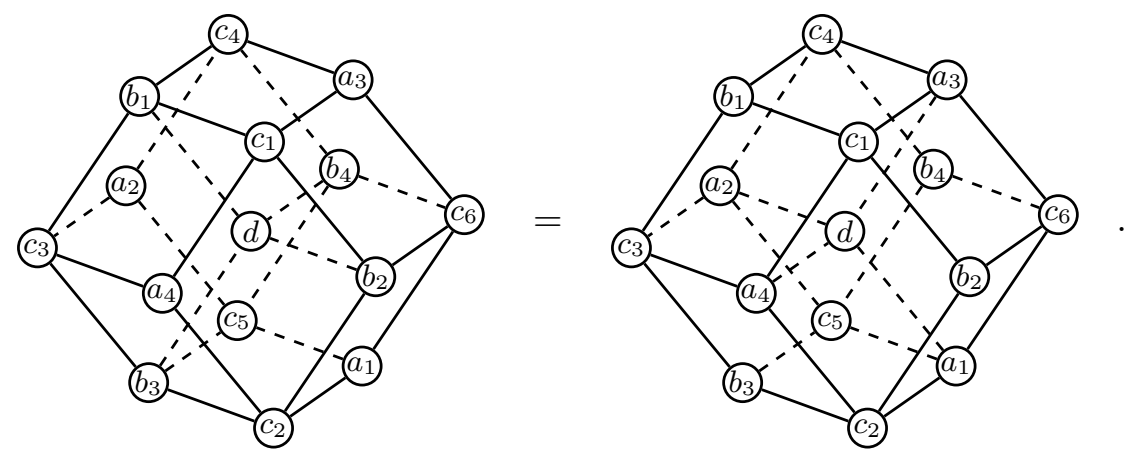

\section{$34 \mathrm{~d} \mathcal{N}=1$ quiver gauge theories}

Now we wish to apply the above construction to $4 \mathrm{~d} \mathcal{N}=1$ quiver gauge theories realized by brane box configurations. In this section, we explain how the elements that entered the construction - TQFT, lattice of line operators, and extra dimensions - arise nicely in the supersymmetric indices of brane box models. Furthermore, we determine the associated integrable lattice model, and demonstrate that the Yang-Baxter equation for this model is equivalent to the equality between the supersymmetric index of $\mathrm{SU}(N) \mathrm{SQCD}$ with $2 N$ flavors and that of its Seiberg dual.

\subsection{Brane box models}

Consider Type IIB superstring theory, and suppose that we have $N$ coincident D5-branes extending in the directions 012346, and a number of NS5-branes in 012345 and 012367 :

\begin{tabular}{|l|cccccccccc|}
\hline & 0 & 1 & 2 & 3 & 4 & 5 & 6 & 7 & 8 & 9 \\
\hline D5 & $\times$ & $\times$ & $\times$ & $\times$ & $\times$ & & $\times$ & & & \\
NS5 & $\times$ & $\times$ & $\times$ & $\times$ & $\times$ & $\times$ & & & & \\
NS5 & $\times$ & $\times$ & $\times$ & $\times$ & & & $\times$ & $\times$ & & \\
\hline
\end{tabular}

These branes are all located at the same point in the 89-plane. So each NS5-brane intersects with the D5-branes along a straight line parallel to the 4- or 6-axis.

Macroscopically, this brane system realizes a supersymmetric quiver gauge theory, much the same way as the brane constructions of $3 \mathrm{~d} \mathcal{N}=4$ [49] and $4 \mathrm{~d} \mathcal{N}=2$ [11] gauge theories. In those constructions, D3- or D4-branes are suspended between NS5-branes placed at finite intervals in the 6-direction. The difference here is that the D5-branes are divided by NS5-branes into finite segments in the 4-direction as well. The resulting theory therefore has less spacetime dimensions and supersymmetry, but a more complicated quiver: it is a $4 \mathrm{~d} \mathcal{N}=1$ gauge theory described by a planar quiver diagram. Brane box models, first studied in [18], are theories constructed from brane configurations of this kind.

To avoid complications associated with semi-infinite branes, let us compactify the 46plane to a torus $T^{2}$. Then, the intersections of the NS5-branes with the D5-branes form a lattice on the 46-torus. We draw the intersections by oriented dashed lines and call them rapidity lines, anticipating connection with integrable lattice models. We choose the 


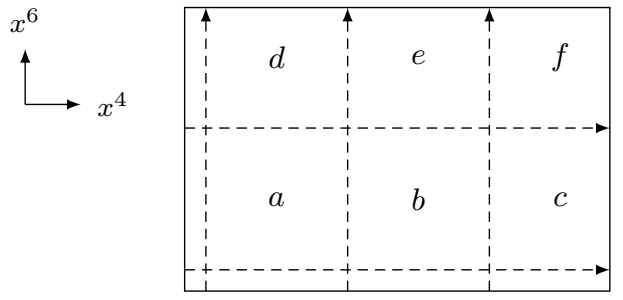

(a)

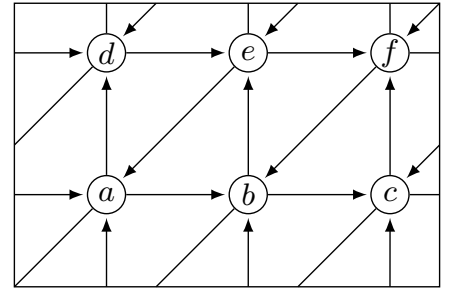

(b)

Figure 2. The $2 \times 3$ brane box model. (a) The configuration of NS5-brane intersections on the 46-torus. (b) The corresponding quiver diagram.

orientations for horizontal lines in such a way that they are parallel (and not antiparallel) to one another, and similarly for vertical lines. See figure 2(a) for an example of a brane box model, in which the rapidity lines make up a $2 \times 3$ lattice.

The rule for determining the quiver is as follows. We label the faces of the lattice by letters $a, b, \ldots$ To the $a$ th face is associated a vector multiplet for gauge group $\mathrm{SU}(N)_{a}$, which originates from open strings whose ends are attached on this part of the D5-branes. Since the rank of the gauge group is fixed throughout our discussion, in our quiver diagram we represent the vector multiplet simply by a circle node labeled $a$ :

From two faces $a$ and $b$ adjacent to each other or sharing a vertex, we get a chiral multiplet in a bifundamental representation of $\mathrm{SU}(N)_{a} \times \mathrm{SU}(N)_{b}$. This is represented by an arrow between the two nodes:

$$
\text { (a) } \longrightarrow \text { (b) }
$$

is a chiral multiplet in the representation $(\overline{\mathbf{N}}, \mathbf{N})$ of $\mathrm{SU}(N)_{a} \times \mathrm{SU}(N)_{b}$, coupled to the vector multiplets. If four faces $a, b, c, d$ are placed around a vertex in the counterclockwise order, the corresponding part of the quiver is given by

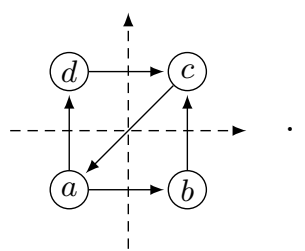

What makes brane box models physically interesting is that they are chiral: between nodes, arrows only point in one direction. The quiver diagram for the $2 \times 3$ brane box model is shown in figure $2(\mathrm{~b})$.

The same diagram also encodes the superpotential of the theory. The quiver consists of a collection of triangles that cover the 46-torus. For each triangle formed by three arrows, there is a cubic superpotential term given by the trace of the product of the corresponding bifundamental multiplets, with sign determined by the orientation of the arrows. The precise form of the superpotential will not be important for our discussion; the quantities we will consider are largely independent of this information. 


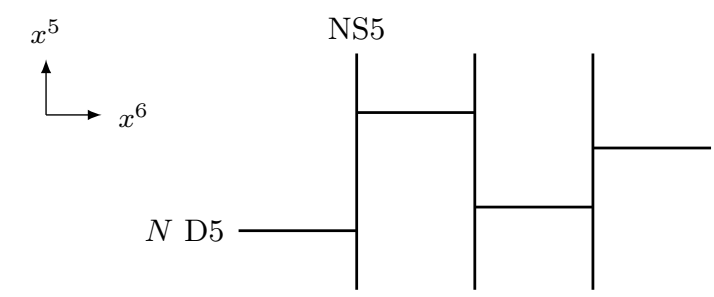

Figure 3. Sliding D5-brane segments along NS5-branes. Illustrated here is an ideal situation with $g_{s}=0$.

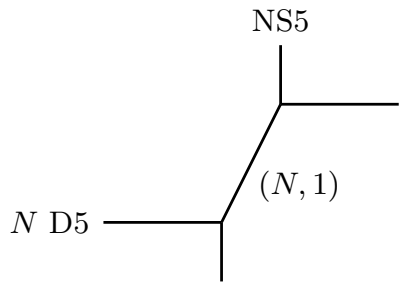

(a)

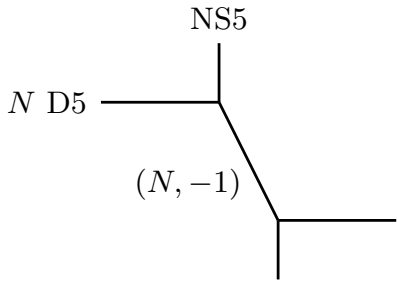

(b)

Figure 4. For $g_{s}>0$, taking apart two D5-brane segments creates either (a) a tilted $(N, 1) 5$-brane or (b) a tilted $(N,-1) 5$-brane.

The gauge group of a brane box model is the product of $\mathrm{SU}(N) \mathrm{s}$ and not $\mathrm{U}(N) \mathrm{s}$. To understand why, let us suppose for simplicity that there are only NS5-branes spanning the 012345 directions. In this case we get a $5 \mathrm{~d} \mathcal{N}=1$ theory. We are taking the string coupling $g_{s}$ to be small, which means that the NS5-branes have tension much larger than that of the D5-branes and can be treated as rigid objects. These heavy NS5-branes "chop" the D5-branes into finite segments in the 6-direction. Imagine sliding these segments slightly in the 5-direction. If $g_{s}$ were strictly zero, the NS5-branes would be infinitely heavier than the D5-branes and not affected by such a deformation at all. Then we would have a situation sketched in figure 3 .

In reality, $g_{s}$ is small but nonzero, and the deformation does affect the NS5-branes. What actually happens is that when a stack of $N$ D5-branes hit on an NS5-brane, these branes combine to form a bound state - an $(N, 1)$ or $(N,-1) 5$-brane, depending on the relative positions of the branes - which then goes off diagonally, as shown in figure 4. As a result, moving the D5-brane segments in the 5-direction entails shifts of semi-infinite NS5branes in the 6 -direction, and this costs an infinite amount of energy. The $x^{5}$-coordinates of the D5-brane segments are therefore frozen. As the diagonal U(1) gauge field on each segment is in the same $5 \mathrm{~d}$ multiplet as the scalar $x^{5}$, it is frozen as well. Thus, the gauge group of the $5 \mathrm{~d}$ theory is the product of $\mathrm{SU}(N) \mathrm{s}$, times a $\mathrm{U}(1)$ factor corresponding to the overall center of mass coordinate of the D5-branes. The $\mathrm{U}(1)$ factor is decoupled from the rest of the dynamics, so we ignore it. 


\subsection{R-symmetry and flavor symmetries}

The brane system (3.1) is invariant under rotations in the 89-plane. The rotational symmetry appears in the effective $4 \mathrm{~d}$ theory as an R-symmetry $\mathrm{U}(1)_{R}$. However, the R-charges of the bifundmanetal multiplets are not determined uniquely by the brane configuration, because of the possibility of shifting them by U(1) flavor charges. This ambiguity plays a crucial role in the connection to integrable lattice models, so let us look at it closely.

We denote by $R_{a b}$ the R-charge of the bifundamental multiplet represented by an arrow going from node $a$ to $b$. There are two conditions for a set of R-charges $\left\{R_{a b}\right\}$ to be realized in a brane box model. The first is that the superpotential must have R-charge 2 so that it preserves $\mathrm{U}(1)_{R}$. Thus, for every triangle

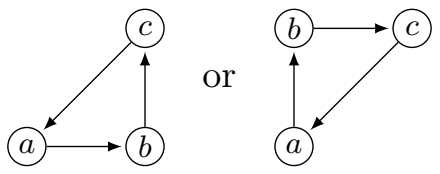

we must have

$$
R_{a b}+R_{b c}+R_{c a}=2 .
$$

The second is that $\mathrm{U}(1)_{R}$ must be nonanomalous. This requirement leads to the condition that at every node, the R-charges of the bifundamental multiplets starting from or ending on that node add up to the number of such multiplets minus two. In our case, for every

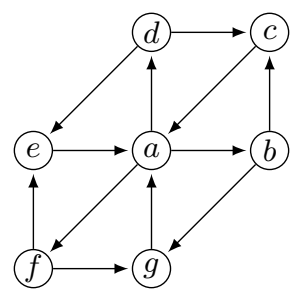

we must have

$$
R_{a b}+R_{c a}+R_{a d}+R_{e a}+R_{a f}+R_{g a}=4 .
$$

The second condition is equivalent to the vanishing of the one-loop $\beta$-functions for the gauge couplings, and one expects that the theory flows to a nontrivial infrared fixed point when this condition is satisfied.

What is the dimension of the space of solutions to the constraint equations? The quiver of a brane box model provides a triangulation of $T^{2}$. The number of R-charges to be assigned is equal to the number of edges $E$, whereas the number of constraints is equal to the number of faces $F$ plus that of vertices $V$. Thus, naive counting would suggest that the dimension is given by minus the Euler characteristic $\chi=V-E+F$, which vanishes for $T^{2}$. This is not the case, though. For example, we can assign R-charge $R=1-r$ to the horizontal arrows, $R=1+r$ to the vertical ones and $R=0$ to the diagonal ones, with $r$ being a free parameter.

The reason for the mismatch is that the constraints are not all independent. Indeed, using the triangle condition (3.6), the anomaly cancellation condition (3.8) can be replaced by

$$
R_{a d}-R_{b c}+R_{g a}-R_{f e}=0 .
$$




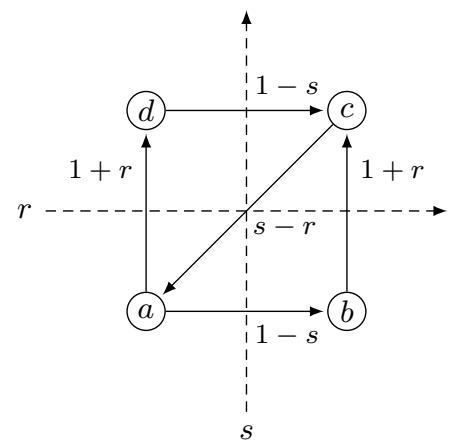

Figure 5. The R-charge parameterization for brane box models.

The sum of the left-hand side, with $a$ running over all nodes in the same row of the quiver, is zero due to the periodicity of $T^{2}$. So there is one relation for each row. Similarly, there is one relation for each column. For an $m \times n$ lattice, the dimension of the space of solutions is therefore at least $m+n$, the total number of rapidity lines.

These $m+n$ degrees of freedom in the R-charge assignment may be thought of as degrees of freedom associated to the rapidity lines. Concretely, we can parametrize the R-charges as follows. To each rapidity line we assign a real parameter, and call it the rapidity or spectral parameter of the rapidity line. Then, an arrow has $R=1-r$ if it is horizontal and $R=1+r$ if vertical, where $r$ is the spectral parameter of the rapidity line intersecting with that arrow. The R-charges of the diagonal arrows are determined by the condition (3.6). The rule is summarized in figure 5. One readily sees that with this rule, the condition (3.8) is also satisfied.

In general, a U(1) R-symmetry can be shifted by U(1) flavor symmetries, that is, global $\mathrm{U}(1)$ symmetries that commute with the supersymmetry algebra. In view of this fact, the degrees of freedom in the R-charge assignment just found suggests that there are $m+n$ $\mathrm{U}(1)$ flavor symmetries, each associated to a single rapidity line.

The origin of these global symmetries can be most clearly understood in the original brane picture. Let us pick an NS5-brane, say the one corresponding to the $\alpha$ th rapidity line, and imagine, as before, slightly sliding the D5-brane segments that end on it from left and right. This operation splits the NS5-brane into the upper and lower semi-infinite NS5branes, joined by a finite segment of $(N, \pm 1) 5$-brane; see figure 4 . For definiteness, consider the case with an $(N, 1) 5$-brane. Bifundamental multiplets arise from open strings whose ends are localized at the junctions where semi-infinite NS5-branes meet D5-branes and the $(N, 1) 5$-brane. These strings may be viewed as fundamental strings $((N, 0)$-strings $)$, as in figure 6(a), or D-strings ((0,1)-strings) with the opposite orientation, ${ }^{5}$ as in figure $6(\mathrm{~b})$. As such, they are acted upon by global U(1) gauge transformations on the semi-infinite NS5branes. In the effective $4 \mathrm{~d}$ theory that emerges when the split branes are rejoined, gauge transformations on the upper and lower branes appear as elements in the same symmetry

\footnotetext{
${ }^{5}$ To determine the orientation, we draw an $(N, 0)$-string attached on the D5-branes, and move the endpoints to the semi-infinite NS5-branes. When an endpoint passes a junction, an $(N, 1)$-string is created due to charge conservation. When the other endpoint passes the other junction, the $(N, 1)$-string is annihilated, leaving a $(0,1)$-string. The orientation of the $(0,1)$-string can be seen from the final picture.
} 


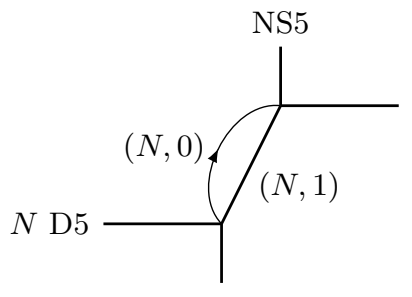

(a)

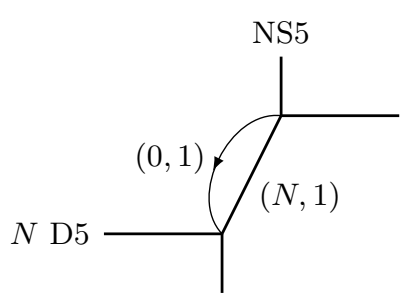

(b)

Figure 6. (a) An ( N,0)-string stretched between the D5-NS5- $(N, 1)$ junctions. (b) The same string may be regarded as a $(0,1)$-string with the opposite orientation.

$\mathrm{U}(1)_{\alpha}$, just acting in opposite manners. Hence, a $\mathrm{U}(1)$ flavor symmetry is associated to each NS5-brane or rapidity line.

Equivalently, we may regard $\mathrm{U}(1)_{\alpha}$ as arising from the $\mathrm{U}(1)$ gauge symmetries on the D5-brane segments, which we have seen are nondynamical. From this point of view, $\mathrm{U}(1)_{\alpha}$ acts on all D5-brane segments on the "right" side of the $\alpha$ th N5-brane, or on all of those on the "left" side in the opposite manner. Of course, the words "left" and "right" are not really meaningful when the 4- and 6-direction are periodic, but we can make sense of this interpretation by, roughly speaking, considering the D5-branes to be sections of a $\mathrm{U}(1)_{\alpha}$-bundle on the 46 -torus.

This point is probably easier to understand if we consider the situation where there are only NS5-branes in the 012345 directions, and look at the mass parameter $m_{\alpha}$ associated to $\mathrm{U}(1)_{\alpha}$ in the corresponding $5 \mathrm{~d} \mathcal{N}=1$ theory. $m_{\alpha}$ is the value of the real scalar in the background vector multiplet for $\mathrm{U}(1)_{\alpha}$, and proportional to the difference between the $x^{5}$ coordinates of the two D5-brane segments that end on the $\alpha$ th NS5-brane. The periodicity in the 6-direction suggests that the sum of $m_{\alpha}$ over the rapidity lines must vanish. However, this constraint can be modified if we compactify the 6-direction using a twisted boundary condition that involves a shift in the 5 -direction. (The same twisted boundary condition is used in the brane construction of $4 \mathrm{~d} \mathcal{N}=2$ elliptic models [11].) This modification introduces one more parameter, namely the amount of shift, leading to the same number of mass parameters as the rapidity lines.

We normalize the $\mathrm{U}(1)_{\alpha}$-charge $F_{\alpha}$ in such a way that horizontal and vertical bifundamental arrows intersecting with the $\alpha$ th rapidity line have $F_{\alpha}=-1$ and +1 , respectively, while $F_{\beta}=0$ for $\beta \neq \alpha$. The charges of the diagonal arrows are found by demanding that the superpotential have $F_{\alpha}=0$ for all $\alpha$. Using $F_{\alpha}$, our R-charge parametrization can be concisely written as

$$
R=R_{0}+\sum_{\alpha} r_{\alpha} F_{\alpha}
$$

where $R_{0}$ is the $\mathrm{R}$-charge defined by assigning $R_{0}=1$ to the horizontal and vertical arrows, and $r_{\alpha}$ is the parameter of the $\alpha$ th rapidity line. As expected, the degrees of freedom in the definition of $R$ come from shifts by flavor symmetries.

We have seen above that there are at least $m+n \mathrm{U}(1)$ flavor symmetries, but have not determined the precise number. Actually, in some cases there are extra U(1) symmetries in 


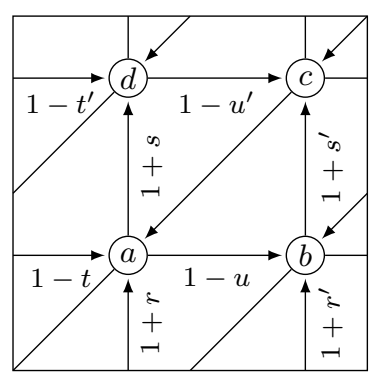

Figure 7. The general R-charge assignment for the $2 \times 2$ brane box model. Here only the Rcharges of the horizontal and vertical arrows are indicated, and the variables satisfy the relation $r^{\prime}-r=s-s^{\prime}=t-t^{\prime}=u^{\prime}-u$.

addition to the ones discussed above. For example, for a $2 \times 2$ lattice, the most general Rcharge assignment has five parameters rather than four, as shown in figure 7 . In fact, from the relation between the brane box and brane tiling constructions explained in section 4 , one can deduce that the number of $\mathrm{U}(1)$ flavor symmetries is equal to $m+n+\operatorname{gcd}(m, n)-1$. We will not consider these extra flavor symmetries. Alternatively, one may assume that $m$ and $n$ are coprime.

\subsection{Structure of a TQFT with extra dimension}

So far we have described the brane box configuration (3.1) from the point of view of the effective $4 \mathrm{~d} \mathcal{N}=1$ theory. To make contact with the framework discussed in section 2, let us instead think of it as realizing 6d maximally supersymmetric Yang-Mills theory on the D5-branes, in the presence of codimension-1 defects created by the NS5-branes. We wish to turn this $6 \mathrm{~d}$ theory into a theory that is topological on the 46-torus, and regard the defects as line operators with spectral parameter in the TQFT. This is achieved by applying an appropriate topological twist and restricting physical quantities to compute, as we now explain.

Type IIB superstring theory has $\mathcal{N}=(2,0)$ supersymmetry in $9+1$ dimensions, generated by Majorana-Weyl spinors $\epsilon_{L}, \epsilon_{R}$ such that

$$
\Gamma_{0123456789} \epsilon_{L}=\epsilon_{L}, \quad \Gamma_{0123456789} \epsilon_{R}=\epsilon_{R}
$$

Here $\Gamma_{M}, M=1, \ldots, 9$ are Gamma matrices, and $\Gamma_{0123456789}=\Gamma_{0} \cdots \Gamma_{9}$ is the chirality operator. The D5-branes preserve supersymmetry generated by $\epsilon_{L}, \epsilon_{R}$ satisfying

$$
\epsilon_{L}=\Gamma_{012346} \epsilon_{R}
$$

Likewise, the NS5-branes impose conditions

$$
\epsilon_{L}=\Gamma_{012345} \epsilon_{L}, \quad \epsilon_{R}=-\Gamma_{012345} \epsilon_{R}
$$

and

$$
\epsilon_{L}=\Gamma_{012367} \epsilon_{L}, \quad \epsilon_{R}=-\Gamma_{012367} \epsilon_{R}
$$


on the preserved supersymmetry. The conditions on $\epsilon_{R}$ coming from the NS5-branes actually follow from those on $\epsilon_{L}$ and the D5-brane condition, hence are redundant. Since $\Gamma_{012345}$ and $\Gamma_{012367}$ square to the identity and are traceless, half of their eigenvalues are +1 and the other half are -1 . Moreover, they commute with each other and are simultaneously diagonalizable. Each NS5-brane condition therefore halves the number of independent solutions for $\epsilon_{L}$. Given $\epsilon_{L}$, the D5-brane condition determines $\epsilon_{R}$. In total, the 5-branes preserve four supercharges, which generate the $\mathcal{N}=1$ supersymmetry of the $4 \mathrm{~d}$ theory we have been studying.

From the NS5-brane conditions, we see $\Gamma_{45} \epsilon_{L}=\Gamma_{67} \epsilon_{L}$, or

$$
\left(\Gamma_{46}+\Gamma_{57}\right) \epsilon_{L}=0 .
$$

This equations says that if we replace the rotation group $\mathrm{SO}(2)_{46}$ of the 46-plane (which we decompactify for a moment) with the diagonal subgroup $\mathrm{SO}(2)_{46}^{\prime}$ of $\mathrm{SO}(2)_{46} \times \mathrm{SO}(2)_{57}$, then the preserved supercharges become scalars under the new rotation group. From the same equation we also find that the NS5-brane conditions on $\epsilon_{L}$ can be generalized to

$$
\epsilon_{L}=\Gamma_{0123}\left(\Gamma_{4} \cos \theta+\Gamma_{6} \sin \theta\right)\left(\Gamma_{5} \cos \theta+\Gamma_{7} \sin \theta\right) \epsilon_{L},
$$

where $\theta$ is any angle. This shows that the NS5-branes can be rotated in the 46- and 57plane by the same angle $\theta$ without destroying the supercharges, or for that matter, they can intersect with the 46-plane along arbitrary closed curves as long as the slopes are correlated between the 46- and 57-plane.

From the point of view of the $6 \mathrm{~d}$ theory on the D5-branes, the replacement of $\mathrm{SO}(2)_{46}$ with $\mathrm{SO}(2)_{46}^{\prime}$ is a topological twist along the 46-plane. After the twisting, eight out of the sixteen supercharges are scalars on the 46-plane, and as such, can be preserved even when the 46-plane is replaced by a general 2-manifold $\Sigma$. Codimension- 1 defects can be inserted along arbitrary closed curves on $\Sigma$ while preserving four of the eight supercharges. These defects carry a spectral parameter, as we have seen from the $4 \mathrm{~d}$ point of view.

Now, to produce a desired TQFT from this twisted theory, we simply restrict ourselves to quantities that are independent of the metric on $\Sigma$, and depend on the codimension- 1 defects inserted on $\Sigma$ only through their topology and spectral parameters. Then, the twisted theory becomes a TQFT on $\Sigma$, and the defects become line operators with spectral parameter in this TQFT.

A good example of such a topological quantity is the supersymmetric index of the corresponding $4 \mathrm{~d} \mathcal{N}=1$ theory [50-52]. As the theory has the R-symmetry $\mathrm{U}(1)_{R}$, it can be placed on the Euclidean spacetime $S^{3} \times S^{1}$ without breaking supersymmetry [50, 52, 53]. Under (the double cover of) the isometry group $\mathrm{SU}(2) \times \mathrm{SU}(2)$ of $S^{3}$, the four supercharges transform as a pair of doublets $(\mathbf{2}, \mathbf{1})$. Let $J_{i}, J_{i}^{\prime}$ be the generators of the $\mathrm{SU}(2)$ factors. The supersymmetric index is defined by

$$
\mathcal{I}\left(p, q,\left\{u_{\alpha}\right\} ;\left\{r_{\alpha}\right\}\right)=\operatorname{Tr}_{S^{3}}\left((-1)^{F} p^{J_{3}+J_{3}^{\prime}-R / 2} q^{J_{3}-J_{3}^{\prime}-R / 2} \prod_{\alpha} u_{\alpha}^{F_{\alpha}}\right),
$$

where $p, q, u_{\alpha}$ are complex parameters, and the trace is taken over the Hilbert space on $S^{3}$. This quantity is topological for the following reason. Bosonic and fermionic states 
with energy $E \neq 2 J_{3}-R$ are paired by the action of a supercharge. (Here we are taking the radius of $S^{3}$ to be 1.) Due to the presence of the factor $(-1)^{F}$, pairwise cancellations occur and the only contributions to the trace come from states with $E=2 J_{3}-R$. Under continuous changes of the parameters of the theory (other than the rapidities $r_{\alpha}$ on which $R$ implicitly depends), the index remains invariant since states can be brought into or out of the right energy level only in boson-fermion pairs. The index is therefore protected against such changes, in particular against deformations of geometric parameters such as the shapes of line operators and the metric on $\Sigma$.

There are other topological quantities. For example, we can replace the $S^{3}$ in the above definition of the supersymmeric index with $S^{2} \times S^{1}$ or a lens space $L(p, q)$ [54]. Unfortunately, though, an explicit expression for the index is not known except for simple cases such as $L(p, 1)$ [15]. We will focus on the $S^{3}$ index, for which the relevant mathematical results are available.

So we have obtained from brane box models a $2 \mathrm{~d}$ TQFT that has a family of line operators with spectral parameter. We can now apply the construction discussed in section 2 and get a vertex model, by placing the TQFT on $T^{2}$ and making a lattice of line operators. However, we cannot tell whether the model is integrable just from the structure of a TQFT. For the integrability, it is necessary to show, in addition, that no phase transitions occur when two line operator are untangled or a line operator passes through the intersection of two other line operators. In Costello's argument, these properties are guaranteed by the existence of extra dimensions in which the line operators can miss one another.

We can make a similar argument in the present case. Let us go back to the brane picture. To the configuration (3.1), we apply T-duality in the 3-direction (which we take to be the $S^{1}$-direction of the spacetime $S^{3} \times S^{1}$ ) to convert the D5-branes to D4-branes, and then lift the resulting Type IIA brane system to M-theory:

\begin{tabular}{|c|ccccccccccc|}
\hline & 0 & 1 & 2 & 3 & 4 & 5 & 6 & 7 & 8 & 9 & 10 \\
\hline M5 & $\times$ & $\times$ & $\times$ & & $\times$ & & $\times$ & & & & $\times$ \\
M5 & $\times$ & $\times$ & $\times$ & $\times$ & $\times$ & $\times$ & & & & & \\
M5 & $\times$ & $\times$ & $\times$ & $\times$ & & & $\times$ & $\times$ & & & \\
\hline
\end{tabular}

These dualities transform the $6 \mathrm{~d}$ theory on the D5-branes into an $\mathcal{N}=(2,0)$ theory on a stack of M5-branes, and the codimension-1 defects into codimension-2 defects. There is now an extra dimension along which line operators can move, namely the M-theory circle. Its presence ensures the integrability of the vertex model.

The similarity to Costello's argument suggests that the $x^{10}$-coordinates of line operators are related to the spectral parameters $r_{\alpha}$. This is indeed true, as the following analysis shows.

If we trade off the flavor fugacities $u_{\alpha}$ for new parameters $s_{\alpha}$ given by $u_{\alpha}=(p q)^{-s_{\alpha} / 2}$, the supersymmetric index (3.17) depends on $s_{\alpha}$ only through the combination $R+\sum_{\alpha} s_{\alpha} F_{\alpha}$. Hence, the effect of turning on nonzero values for $s_{\alpha}$ is equivalent to shifting $r_{\alpha} \rightarrow r_{\alpha}+s_{\alpha}$, and $s_{\alpha}$ represent the same degrees of freedom as $r_{\alpha}$. We want to understand how $s_{\alpha}$ are described in the language of branes, and keep track of them under the dualities. 
As we saw before by considering the deformation depicted in figure $6, \mathrm{U}(1)_{\alpha}$ can be interpreted as either the global gauge symmetry on the lower half of the $\alpha$ th NS5-brane, or that on the upper half acting in the opposite manner. The conserved current $J_{\alpha}$ for $\mathrm{U}(1)_{\alpha}$ is therefore supported along $C_{\alpha}$ on the 46-torus, and couples to the $6 \mathrm{~d}$ theory through a term of the form

$$
i \int_{S^{3} \times S^{1} \times T^{2}}\left(A_{\alpha}^{-}-A_{\alpha}^{+}\right) \wedge \star J_{\alpha} .
$$

Here $A_{\alpha}^{-}$and $A_{\alpha}^{+}$are the gauge fields on the lower and upper halves of the NS5-brane, restricted to the brane intersection; they are related there to the $\mathrm{U}(1)$ parts of the gauge fields on the D5-brane segments by some boundary condition. We give a background value to the gauge fields such that $A_{\alpha}^{-}-A_{\alpha}^{+}$is constant along $S^{3}$ and $C_{\alpha}$. As $F_{\alpha}$ is the integral of $\star J_{\alpha}$ over $S^{3}$ and $T^{2}$, we find that the above term becomes

$$
i \int_{S^{1}}\left(A_{\alpha}^{-}-A_{\alpha}^{+}\right) F_{\alpha}
$$

This term enters the path integral as an exponentiated factor, so $s_{\alpha}$ is proportional to the difference between the holonomies of $A_{\alpha}^{-}$and $A_{\alpha}^{+}$around the 3 -circle.

These holonomies are mapped under the T-duality to the background values of a scalar field on the upper and lower halves of the corresponding Type IIA NS5-brane. Upon lifting to M-theory, the NS5-brane becomes an M5-brane, and the scalar is mapped to the $x^{10}$ coordinate. Therefore, the spectral parameter for a line operator is given by the difference between the $x^{10}$-coordinates of the upper and lower halves of the M5-brane.

We remark that if the holonomies of $A_{\alpha}^{+}$and $A_{\alpha}^{-}$are equal, then the whole $\alpha$ th NS5brane is mapped to a single point on the M-theory circle, and the supersymmetric index does not depend on its position at all. This observation is consistent with the fact that the $\mathcal{N}=(2,0)$ theory compactified on $S^{3}$ has a topological sector that is equivalent to Chern-Simons theory with complex gauge group at level $k=1$ [40]. In the present setting, the NS5-brane creates a Wilson line in the Chern-Simons theory on $T^{2} \times S^{1}$, and its precise position on the 3-manifold is irrelevant. When the holonomies are different, the Wilson line splits into two line operators, and apparently they no longer belong to the topological sector. It is clear from our viewpoint that the same consideration should apply to other indices obtained by replacing $S^{3}$ with appropriate 3 -manifolds. Indeed, the $\mathcal{N}=(2,0)$ theory on $S^{2} \times S^{1}$ has a topological sector equivalent to complex Chern-Simons theory at level $k=0[38,39]$, while the lens space $L(k, 1)$ gives complex Chern-Simons theory at level $k$ [43]. Similarly, a twisted product of $\mathbb{R}^{2}$ and $S^{1}$ leads to analytically continued ChernSimons theory, which is the holomorphic sector of complex Chern-Simons theory [37, 55-57].

\subsection{Integrable lattice model for brane box models}

Finally, let us identify the integrable lattice model associated with brane box models. To do so, we look at the integral formula for the supersymmetric index.

Recall that the flavor fugacities $u_{\alpha}$ represent the same degrees of freedom as the spectral parameters $r_{\alpha}$. Since we are going to keep the dependence on $r_{\alpha}$, we set $u_{\alpha}=1$. With this 
understood, the supersymmetric index of a brane box model is computed as follows [50, 58]. First, we assign the factor

$$
\mathcal{I}_{\mathrm{V}}\left(z_{a}\right)=\left((p ; p)_{\infty}(q ; q)_{\infty}\right)^{N-1} \prod_{i \neq j} \frac{1}{\Gamma\left(z_{a, i} / z_{a, j} ; p, q\right)}
$$

to the vector multiplet represented by node $a$, and

$$
\mathcal{I}_{\mathrm{B}}\left(z_{a}, z_{b} ; R_{a b}\right)=\prod_{i, j} \Gamma\left((p q)^{R_{a b} / 2} z_{b, j} / z_{a, i} ; p, q\right)
$$

to the bifundamental chiral multiplet with $R=R_{a b}$ represented by an arrow from node $a$ to $b$. Here

$$
\Gamma(z ; p, q)=\prod_{i, j=0}^{\infty} \frac{1-z^{-1} p^{i+1} q^{j+1}}{1-z p^{i} q^{j}}
$$

is the elliptic Gamma function, $(z ; q)_{\infty}=\prod_{i=0}^{\infty}\left(1-z q^{i}\right)$, and $z_{a}$ collectively denotes the fugacities $z_{a, i}, i=1, \ldots, N$ for the gauge group $\mathrm{SU}(N)_{a}$, obeying $\prod_{i=1}^{N} z_{a, i}=1$. Then, we multiply the factors from all nodes and arrows, and integrate over the fugacities:

$$
\mathcal{I}=\oint \prod_{(a)} \frac{1}{N !} \prod_{j=1}^{N-1} \frac{\mathrm{d} z_{a, j}}{2 \pi i z_{a, j}} \mathcal{I}_{\mathrm{V}}\left(z_{a}\right) \prod_{(a) \rightarrow(b)} \mathcal{I}_{\mathrm{B}}\left(z_{a}, z_{b} ; R_{a b}\right)
$$

The integration for each fugacity is performed over the unit circle $\left\{\left|z_{a, i}\right|=1\right\}$.

The above procedure clearly shows the statistical mechanical nature of the supersymmetric index. In the statistical mechanical interpretation, $z_{a, i}$ are continuous state variables, and the factors $\mathcal{I}_{\mathrm{V}}$ and $\mathcal{I}_{\mathrm{B}}$ are the Boltzmann weights for self- and nearestneighbor interactions, respectively. The partition function is defined by multiplying the weights and summing over all possible state configurations, and this is identified with the supersymmetric index.

Since the state variables $z_{a, i}$ are assigned to the nodes of the quiver which correspond to the faces of the lattice on the 46-torus, it is natural to formulate this model as an IRF model. We can take its Boltzmann weight to be ${ }^{6}$

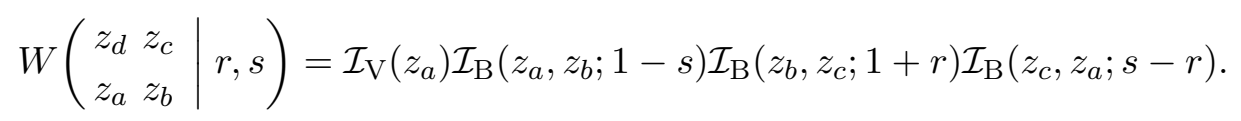

\footnotetext{
${ }^{6}$ This is not the only choice. For example, one may replace the right-hand side with the more symmetric expression

$$
\begin{aligned}
\sqrt{\mathcal{I}_{\mathrm{V}}\left(z_{a}\right) \mathcal{I}_{\mathrm{B}}\left(z_{a}, z_{b} ; 1-s\right) \mathcal{I}_{\mathrm{B}}\left(z_{b}, z_{c} ; 1+\right.} & +r) \mathcal{I}_{\mathrm{B}}\left(z_{c}, z_{a} ; s-r\right) \\
& \times \sqrt{\mathcal{I}_{\mathrm{V}}\left(z_{c}\right) \mathcal{I}_{\mathrm{B}}\left(z_{c}, z_{a} ; s-r\right) \mathcal{I}_{\mathrm{B}}\left(z_{a}, z_{d} ; 1+r\right) \mathcal{I}_{\mathrm{B}}\left(z_{d}, z_{c} ; 1-s\right)}
\end{aligned}
$$
}

without spoiling the integrability. 
Graphically, it can be represented as

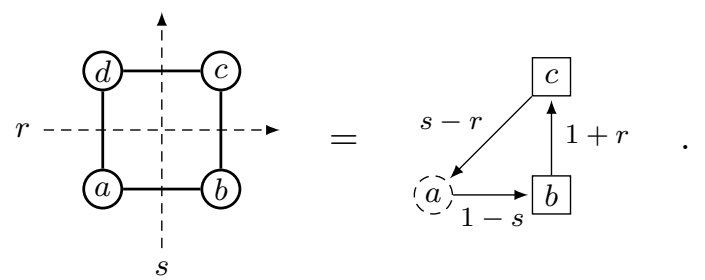

(Notice a slight discrepancy in the notation compared to the original definition (2.18). Here $a, b$, etc. on the left-hand side really stands for $z_{a}, z_{b}$, etc.) A square node $a$ indicates that the associated factor $\mathcal{I}_{\mathrm{V}}\left(z_{a}\right)$ is absent, and a node $(\bar{a})$ drawn with a dashed line means that the factor is present but integration is not performed over $z_{a}$. The intersections of NS5-branes with the D5-branes, which create line operators in the TQFT on the 46-torus, play the roles of rapidity lines, as anticipated.

Let us show that this Boltzmann weight satisfies the Yang-Baxter equation (2.19). For this purpose we will need the identity

$$
\mathcal{I}_{\mathrm{B}}\left(z_{a}, z_{b} ; R_{a b}\right) \mathcal{I}_{\mathrm{B}}\left(z_{b}, z_{a} ; R_{b a}\right)=1
$$

which holds for $R_{a b}+R_{b a}=2$. In effect, it means that we can erase two arrows going in opposite directions between the same pair of nodes:

$$
a=b \quad b \quad b
$$

This property reflects the fact that if one has chiral multipets $\Phi_{1}, \Phi_{2}$ in conjugate representations whose $\mathrm{R}$-charges add up to 2 , one can give them masses by turning on the superpotential term $m \Phi_{1} \Phi_{2}$. The supersymmetric index is independent of $m$, and in the limit $m \rightarrow \infty$, these multiplets become infinitely massive and decouple, leaving no contribution to the index.

Now, plugging the expression (3.27) into the Yang-Baxter equation (2.19), we find

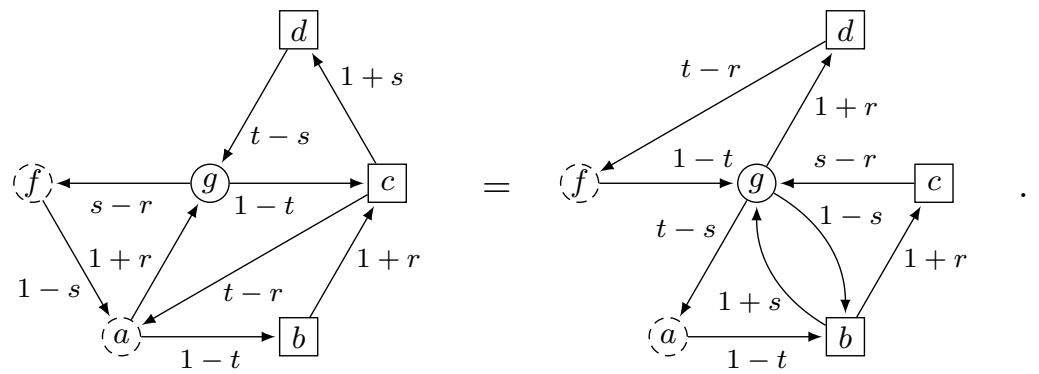

Some nodes and arrows cancel out between the two sides, while the arrows between nodes $b$ and $g$ on the right-hand side cancel thanks to the identity (3.29). Moving the arrows that are not connected to node $g$ from the left- to right-hand side using the same identity, 
we end up with the equality

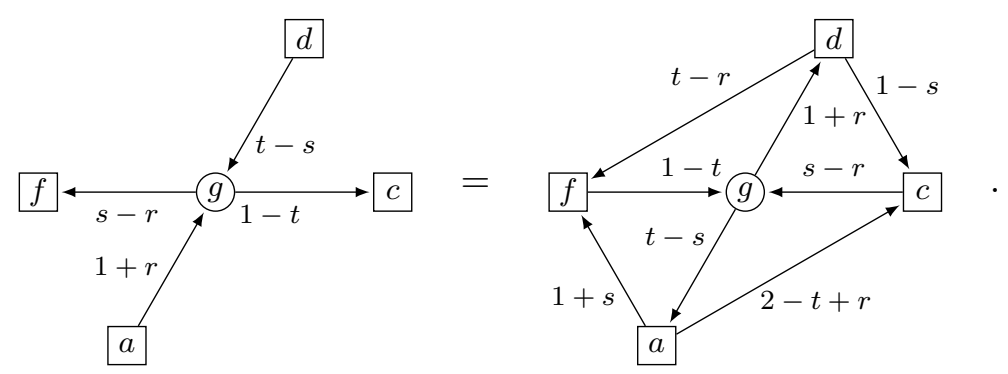

Interestingly, this equality expresses precisely the invariance of the supersymmetric index under Seiberg duality.

Seiberg duality says that for $N_{f}>N_{c}+1$, an $\mathcal{N}=1 \mathrm{SU}\left(N_{c}\right)$ gauge theory with $N_{f}$ flavors $(Q, \widetilde{Q})$ and no superpotential has a dual description in the infrared as an $\mathcal{N}=1$ $\mathrm{SU}\left(N_{f}-N_{c}\right)$ gauge theory with $N_{f}$ flavors $(q, \tilde{q})$ and $N_{f}^{2}$ singlets $M$, coupled through a superpotential $W=\tilde{q} M q$. For both theories, the flavor symmetry is $\mathrm{SU}\left(N_{f}\right)_{L} \times \mathrm{SU}\left(N_{f}\right)_{R} \times$ $\mathrm{U}(1)_{B}$ and the R-symmetry is $\mathrm{U}(1)_{R}$. Under these symmetries and the gauge group $G=$ $\mathrm{SU}\left(N_{c}\right)$ or $\mathrm{SU}\left(N_{f}-N_{c}\right)$, the chiral multiplets transform as follows:

\begin{tabular}{|l|ccccc|}
\hline & $G$ & $\mathrm{SU}\left(N_{f}\right)_{L}$ & $\mathrm{SU}\left(N_{f}\right)_{R}$ & $\mathrm{U}(1)_{B}$ & $\mathrm{U}(1)_{R}$ \\
\hline$Q$ & $\square$ & $\square$ & $\mathbf{1}$ & 1 & $1-N_{c} / N_{f}$ \\
$\widetilde{Q}$ & $\square$ & $\mathbf{1}$ & $\square$ & -1 & $1-N_{c} / N_{f}$ \\
\hdashline$q$ & $\square$ & $\square$ & $\mathbf{1}$ & $N_{c} /\left(N_{f}-N_{c}\right)$ & $N_{c} / N_{f}$ \\
$\tilde{q}$ & $\square$ & $\mathbf{1}$ & $\square$ & $-N_{c} /\left(N_{f}-N_{c}\right)$ & $N_{c} / N_{f}$ \\
$M$ & $\mathbf{1}$ & $\square$ & $\square$ & 0 & $2\left(1-N_{c} / N_{f}\right)$ \\
\hline
\end{tabular}

In the case at hand, $N_{f}=2 N_{c}=2 N$ and the charged chiral multiplets all have $R=1 / 2$. This corresponds to the situation with $(r, s, t)=(-1 / 2,0,1 / 2)$. As we stressed, however, we can shift the R-charge by U(1) flavor charges. The quivers that appear in the equality (3.31) split the $2 N$ flavors into a pair of $N$ flavors. This breaks each of $\mathrm{SU}(2 N)_{L}$ and $\mathrm{SU}(2 N)_{R}$ down to an $\mathrm{SU}(N) \times \mathrm{SU}(N) \times \mathrm{U}(1)$ subgroup. So in total there are three $\mathrm{U}(1)$ flavor symmetries that can be used to shift the R-charge, and they give rise to the three parameters $r, s$ and $t$.

Since the supersymmetric index is protected from continuous changes of the parameters of the theory, it is invariant under renormalization group flow and therefore under Seiberg duality. Indeed, as observed in [58], the invariance under Seiberg duality is equivalent to an identity for the elliptic Gamma function proved in [59]. ${ }^{7}$ Thus, we have demonstrated the integrability of our model. ${ }^{8}$

\footnotetext{
${ }^{7}$ Historically, the identity was first discovered for $N_{c}=2$ and $N_{f}=3$ in [60]. Subsequently it was proved for $N_{c}=2$ and $N_{f}=4$ in [61] and extended to the general case in [59].

${ }^{8}$ The invertibility of the R-matrix follows from the identity

$$
\frac{1}{N !} \oint \prod_{j=1}^{N-1} \frac{\mathrm{d} z_{c, j}}{2 \pi i z_{c, j}} \mathcal{I}_{\mathrm{B}}\left(z_{a}, z_{c} ; R\right) \mathcal{I}_{\mathrm{V}}\left(z_{c}\right) \mathcal{I}_{\mathrm{B}}\left(z_{c}, z_{b} ;-R\right) \mathcal{I}_{\mathrm{V}}\left(z_{b}\right)=K \sum_{\sigma \in S_{N}} \delta\left(z_{a}, \sigma\left(z_{b}\right)\right)
$$




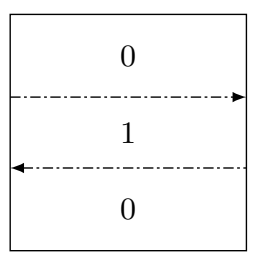

(a)

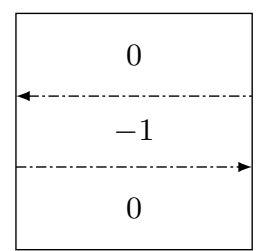

(b)

Figure 8. The 46-space representations for the brane configurations in figure $4(\mathrm{a})$ and $4(\mathrm{~b})$. The number $q$ labeling a region indicates that there is an $(N, q) 5$-brane on that region.

The appearance of Seiberg duality as an integrability condition might be unexpected from the point of view of lattice models. From our perspective, this is not entirely surprising. The integrability is simply a consequence of the topological invariance of the relevant TQFT with extra dimension that has line operators, and these line operators are created by NS5-branes. It is well known that in the brane construction of $4 \mathrm{~d} \mathcal{N}=1$ gauge theories, Seiberg duality is realized by rearrangement of NS5-branes [63, 64].

\section{Brane tiling models}

In the discussion of brane box models in the previous section, we found it helpful to consider a deformation of the brane configuration that splits the D5-branes across NS5-branes and creates $(N, \pm 1) 5$-branes, as in figure 4 . The bifundmantal multiplets represented by the horizontal and vertical arrows in the quiver originate from open strings whose ends are attached on the brane junctions. The original configuration is recovered in the limit where the D5-brane segments are joined together and the $(N, \pm 1) 5$-branes disappear, and these multiplets become massless in this limit.

What happens if we split the D5-branes across every NS5-brane, and keep the distances between the segments finite? In such a situation, the horizontal and vertical multiplets are all massive and absent in the infrared theory. As far as renormalization group invariant quantities such as the supersymmetric index are concerned, these multiplets can therefore be ignored. On the other hand, there are now far more ways in which diagonal multiplets arise. To see this clearly, let us represent brane junctions by oriented dash-dot lines, which we call zigzag paths. The orientation of a zigzag path is such that if it is going up, then there is an $(N, q) 5$-brane on the left and $(N, q+1) 5$-brane on the right for some $q$. For example, the two configurations in figure 4 are represented on the 46 -torus as in figure 8. Diagonal multiplets arise from open strings localized near intersections of rapidity lines or zigzag paths. When the D5-branes split, each rapidity line splits into two antiparallel zigzag paths. Correspondingly, each intersection point breaks apart into four intersection points. As the number of intersection points increases, we expect to get more diagonal multiplets.

proved in [62], where $S_{N}$ is the symmetric group of degree $N$ and the delta function $\delta$ is defined with respect to the measure $\oint \prod_{j=1}^{N-1} \mathrm{~d} z_{a, j} / 2 \pi i z_{a, j}$. The overall factor $\left.K=\prod_{s= \pm 1} \Gamma\left((p q)^{s N R / 2} ; p, q\right)\right)$ can be removed by rescaling the R-matrix. 
The number of $\mathrm{U}(1)$ flavor symmetries also gets larger. Recall that these come from global gauge symmetries on the NS5-branes. In a brane box model, gauge transformations on the upper and lower halves of an NS5-brane give the same flavor symmetry in the $4 \mathrm{~d}$ theory since they simply act in opposite manners on the horizontal and vertical multiplets (and the action on the diagonal multiplets is determined by the requirement that the superpotential be neutral). After the brane configuration is deformed, there are no horizontal or vertical multiplets anymore, so gauge transformations on the two halves can lead to different flavor symmetries.

We can make these statements more precise when the deformed system contains only $(N, 0)$ and $(N, \pm 1) 5$-branes. In fact, in such a situation the system is still described by an $\mathcal{N}=1$ quiver gauge theory [10]. The quiver is such that an $\mathrm{SU}(N)$ node is assigned to each $(N, 0)$ region, and an arrow connects two nodes on regions sharing a vertex:

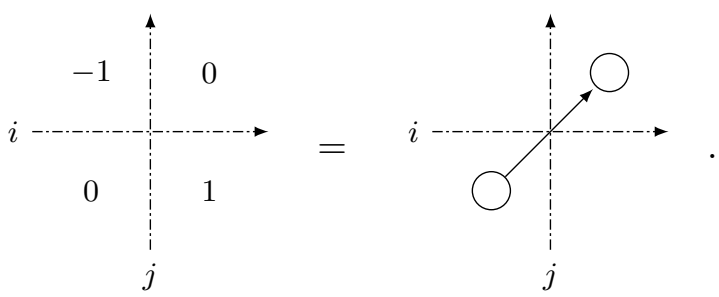

(An $(N, \pm 1) 5$-brane is dual to a single D5-brane, so it supports a $\mathrm{U}(1)$ gauge field which is frozen at low energies.) To the $i$ th zigzag path is associated a flavor symmetry $\mathrm{U}(1)_{i}$, and arrows intersecting with this path have nonzero $\mathrm{U}(1)_{i}$-charge $F_{i}$. We choose the normalization for the $F_{i}$ such that the arrow in the above picture has $F_{i}=+1$ and $F_{j}=-1$. It is known that the $\mathrm{U}(1)_{i}$ account for all nonanomalous $\mathrm{U}(1)$ flavor symmetries present in the gauge theory [65]. They are not independent, however. Since each arrow is intersected precisely by two zigzag paths assigning opposite charges to it, the sum of the charges vanishes:

$$
\sum_{i} F_{i}=0 .
$$

Hence, the number of $\mathrm{U}(1)$ flavor symmetries is equal to the number of zigzag paths minus one.

Once we start thinking in terms of zigzag paths, we can construct many more $\mathcal{N}=1$ theories. We can consider various configurations of zigzag paths, not necessarily obtained by deforming brane box configurations. Such configurations of zigzag paths are called brane tilings. Provided that only $(N, 0)$ and $(N, \pm 1) 5$-branes appear on the graph of zigzag paths, brane tilings yield $\mathcal{N}=1$ quiver gauge theories, whose quivers are determined by the same rule as above. Actually, brane box models can also be described as a brane tiling model; see figure 9 for the brane tiling configuration for the $2 \times 3$ brane box model, whose quiver is shown in figure 2(b). In this sense, brane tilings generalize the brane box construction. The reader may consult the reviews $[66,67]$ for more extensive discussions on brane tilings and their relations to other subjects such as the AdS/CFT correspondence.

Now, let us try to connect brane tilings to an integrable lattice model. Since the only difference compared to the brane box case is the type of defects inserted in the $6 \mathrm{~d}$ 


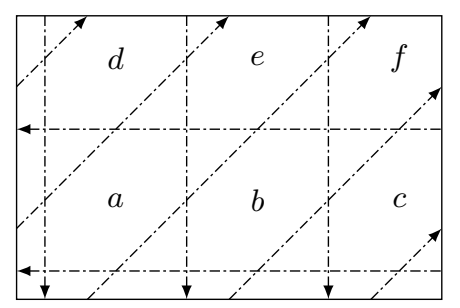

Figure 9 . The $2 \times 3$ brane box model as a brane tiling model.

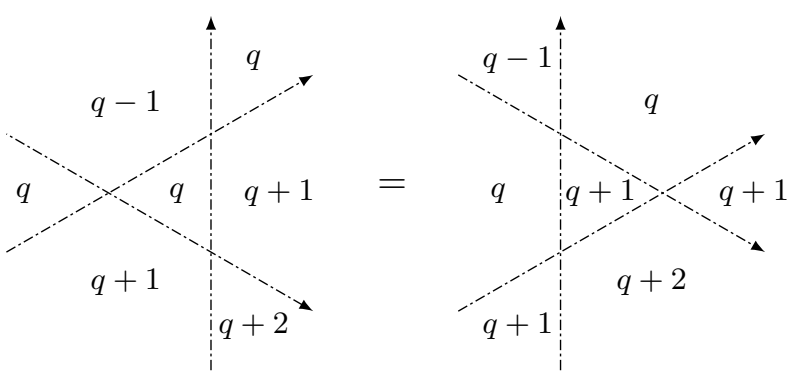

Figure 10. The Yang-Baxter equation for zigzag paths.

theory on the D5-branes, much of the previous argument carries over. Using this $6 \mathrm{~d}$ theory, we can define a $2 \mathrm{~d}$ TQFT whose correlation function for a configuration of zigzag paths is given by the supersymmetric index of the corresponding $4 \mathrm{~d} \mathcal{N}=1$ theory. An extra dimension emerges if we utilize dualities and embed the $6 \mathrm{~d}$ theory in M-theory. Zigzag paths are mapped to semi-infinite M5-branes, and their $x^{10}$-coordinates provide spectral parameters. So we indeed have all ingredients required for the construction of an integrable lattice model. It is reassuring that we have found the relation (4.2), which implies that the supersymmetric index is invariant under an overall shift of the $x^{10}$-coordinates.

The next step would be to write down an integral expression for the supersymmetric index and read off the R-matrix. Here we get into trouble. Clearly, we cannot do this for an arbitrary configuration of zigzag paths; in general, we find $(N, q)$ regions with $|q|>1$, and do not know what the corresponding $4 \mathrm{~d}$ theory is. ${ }^{9}$ Then we are forced to restrict our attention to configurations without such regions. Sadly, this restriction is not compatible with integrability: the Yang-Baxter equation for three zigzag paths always involves regions with $|q|>1$, as can be seen from figure 10 .

The difficulty stems from the fact that zigzag paths are not really line operators. They are more properly called domain walls since they separate regions with different physical properties. This is a problem we did not encounter for brane box models. In that case, rapidity lines were genuine line operators.

\footnotetext{
${ }^{9}$ An exception is the case with $N=2$. In this case, every $(N, q) 5$-brane can be transformed to $(N, 0)$ or $(N, 1) 5$-brane by an $\mathrm{SL}(2 ; \mathbb{Z})$ transformation. So there are only two equivalence classes of 5 -branes, which we can distinguish by painting black or white. This gives a checkerboard pattern on $T^{2}$. Presumably, our construction applied to this case gives an integrable lattice model discovered in [1]. The Yang-Baxter equation for this model first appeared in [68], and is equivalent to an identity proved in [60]. See [69, 70] for more discussions and further developments in this direction.
} 
Our consideration at the beginning of this section suggests a resolution to this problem. We have seen that when a brane box model is deformed to a brane tiling model, a rapidity line splits into two antiparallel zigzag paths. Conversely, we can take two neighboring antiparallel zigzag paths, and regard them as one thick line (or "ribbon") operator. In this way we can make two kinds of line operators, namely

$$
\left(r_{1}, r_{2}\right) \longrightarrow=r_{1} \cdots \cdots \cdots
$$

and another one for which the orientations of the zigzag paths on the right-hand side are flipped. Below we will only use the first one.

If we use this line operator and give the "background charge" $q=-1$ to the TQFT, then regions with $|q|>1$ never arise:

$$
\begin{array}{c|l}
-1 & -1 \\
\hline-1 & -1
\end{array}=\begin{array}{rl|l:l}
-1 & 0 & -1 \\
\hdashline 0 & 1 & 0 \\
\hdashline-1 & 0 & -1
\end{array} .
$$

Thus, a brane tiling constructed with line operators of this type is always described by a quiver gauge theory, and we can readily write down an integral expression for its supersymmetric index. From this expression we can read off the R-matrix of the associated vertex model. Just as in the case for brane box models, we expect that the Yang-Baxter equation for this R-matrix can be understood as an equality between the supersymmetric indices of two dual gauge theories. Let us see if this is the case.

From the quiver rule (4.1), we find that the lattice model in question is a vertex model ${ }^{10}$ with the R-matrix given by

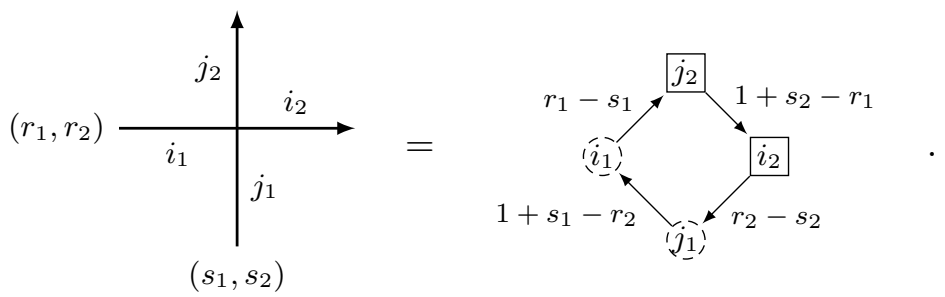

Figure 11 shows the case when the model is placed on a $2 \times 3$ lattice. The R-charges of the bifundamental multiplets, specified by the numbers accompanying the arrows, are chosen to be consistent with the normalization of the flavor charges and add up to 2 around loops. With this choice, the R-charges satisfy the anomaly cancellation condition.

What we have just constructed is the integrable lattice model studied in [2, 8]. (Its generalization to the lens space index was considered in [9].) The Yang-Baxter equation

\footnotetext{
${ }^{10}$ The same model can also be described as an IRF model. However, the IRF description uses line operators of both types, and the Yang-Baxter equation involves twisting of one of the three ribbons. See [9] for a discussion from the IRF viewpoint.
} 


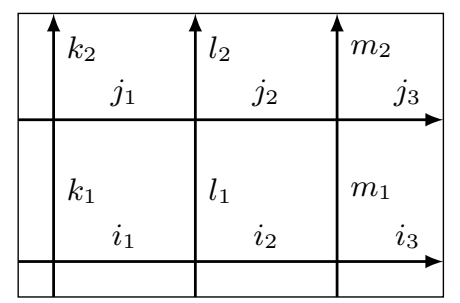

(a)

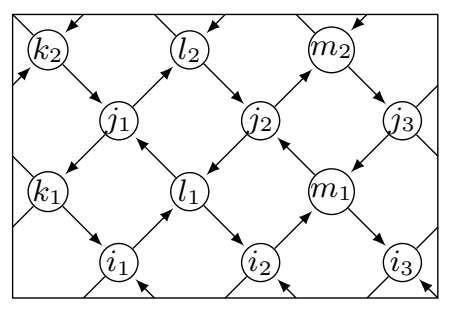

(b)

Figure 11. (a) The $2 \times 3$ lattice model constructed from the line operator (4.3). (b) The quiver of the corresponding gauge theory.

for the model is

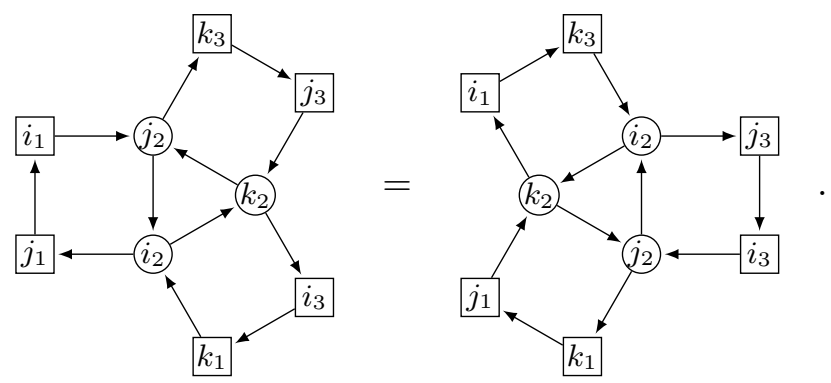

For simplicity we have omitted the R-charges in the quivers. As expected, this equation follows from Seiberg duality: applying the basic duality transformation (3.31) four times, we can turn the left-hand side into the right-hand side. This process is illustrated in figure 12 .

Incidentally, a single Seiberg duality transformation is induced by the Yang-Baxter move involving two zigzag paths and one "twisted" ribbon operator:

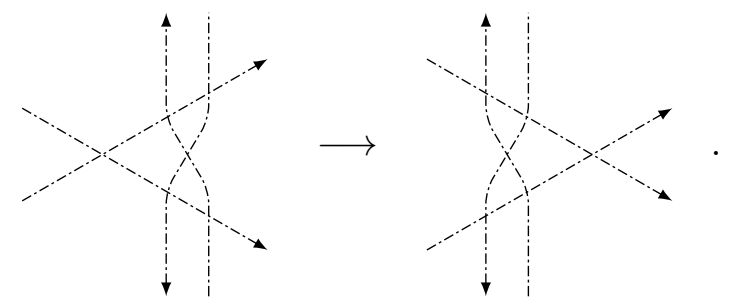

This relation between the Yang-Baxter move and Seiberg duality was pointed out by Hanany and Vegh [71].

\section{$53 \mathrm{~d} \mathcal{N}=2$ and $2 \mathrm{~d} \mathcal{N}=(2,2)$ quiver gauge theories}

The results from the previous sections imply, via T-duality, that there exist similar connections between lower-dimensional quiver gauge theories and integrable lattice models. In this section we discuss integrable lattice models associated with $3 \mathrm{~d} \mathcal{N}=2$ and $2 \mathrm{~d}$ $\mathcal{N}=(2,2)$ theories. We will focus on those coming from brane box configurations, though our analysis can be adapted straightforwardly to the brane tiling case. 

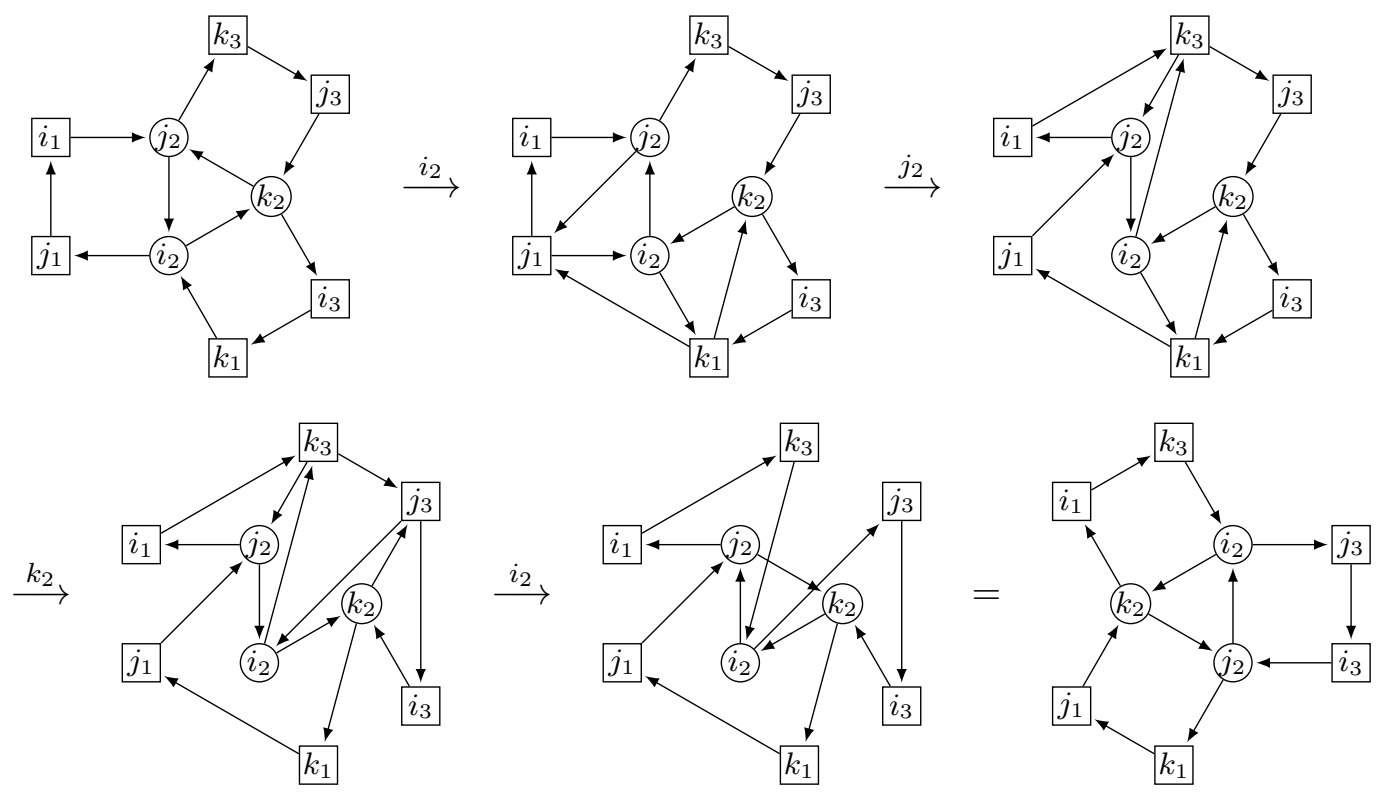

Figure 12. A sequence of duality transformations that demonstrates the Yang-Baxter equation (4.6). The letter on an arrow between two quivers indicates the node at which the transformation is applied. Note that circle nodes can be relabeled freely since their fugacities are integrated over.

\subsection{3d brane box models}

Let us consider the following brane setup in Type IIA superstring theory, which is related to the $4 \mathrm{~d}$ brane box model (3.1) by T-duality along the 2-direction:

\begin{tabular}{|l|cccccccccc|}
\hline & 0 & 1 & 2 & 3 & 4 & 5 & 6 & 7 & 8 & 9 \\
\hline D4 & $\times$ & $\times$ & & $\times$ & $\times$ & & $\times$ & & & \\
NS5 & $\times$ & $\times$ & $\times$ & $\times$ & $\times$ & $\times$ & & & & \\
NS5 & $\times$ & $\times$ & $\times$ & $\times$ & & & $\times$ & $\times$ & & \\
\hline
\end{tabular}

This is a brane box configuration that realizes a $3 \mathrm{~d} \mathcal{N}=2$ quiver gauge theory. Being related by T-duality, the theory is essentially the dimensional reduction of the corresponding $4 \mathrm{~d} \mathcal{N}=1$ theory, and is described by the same quiver (with all Chern-Simons levels vanishing). We expect that the supersymmetric index of this theory is given by the partition function of an integrable lattice model.

As in the $4 \mathrm{~d}$ case, the theory can be placed on $S^{2} \times S^{1}$, with all four supercharges unbroken $[72,73]$. We are interested in the supersymmetric index

$$
\mathcal{I}\left(x ;\left\{r_{\alpha}\right\}\right)=\operatorname{Tr}_{S^{2}}\left((-1)^{F} x^{R+2 J_{3}}\right)
$$

where $J_{3}$ is the Cartan generator of the isometry group $\mathrm{SU}(2)$ of $S^{2}$. It receives contributions only from states with energy $E=R+J_{3}$. As usual, we have omitted the fugacities $u_{\alpha}$ for the flavor symmetries $\mathrm{U}(1)_{\alpha}$, whose effects can be taken into account by keeping the dependence of the R-charge $R$ on the spectral parameters $r_{\alpha}$. 
The 3d supersymmetric index is computed by an integral formula similar to the one in the $4 \mathrm{~d}$ case. The novelty is that there can be nontrivial magnetic fluxes through $S^{2}$. For each gauge group $\mathrm{SU}(N)_{a}$, the flux is specified by a set of integers $m_{a}=\left(m_{a, 1}, \ldots, m_{a, N}\right)$ subject to the condition $\sum_{i} m_{a, i}=0$. Therefore, the formula involves a sum over fluxes as well as an integration over the fugacities for the gauge groups:

$$
\mathcal{I}=\sum_{\left\{m_{a}\right\}} \oint \prod_{(a)} \frac{1}{\left|W_{m_{a}}\right|} \prod_{j=1}^{N-1} \frac{\mathrm{d} z_{a, j}}{2 \pi i z_{a, j}} \mathcal{I}_{\mathrm{V}}\left(z_{a}, m_{a}\right) \prod_{(a) \rightarrow(b)} \mathcal{I}_{\mathrm{B}}\left(\left(z_{a}, m_{a}\right),\left(z_{b}, m_{b}\right) ; R_{a b}\right)
$$

Here $\left|W_{m_{a}}\right|$ is the order of the Weyl group of the subgroup of $G$ that is left unbroken by $m_{a}$, and the vector and bifundamental multiplet factors are given by $[72,74,75]$

$$
\begin{aligned}
& \mathcal{I}_{\mathrm{V}}\left(z_{a}, m_{a}\right)= \prod_{i \neq j} x^{-\left|m_{a, i}-m_{a, j}\right| / 2}\left(1-\frac{z_{a, i}}{z_{a, j}} x^{\left|m_{a, i}-m_{a, j}\right|}\right), \\
& \mathcal{I}_{\mathrm{B}}\left(\left(z_{a}, m_{a}\right),\left(z_{b}, m_{b}\right) ; R_{a b}\right)=\prod_{i, j}\left(x^{1-R_{a b}} \frac{z_{a, i}}{z_{b, j}}\right)^{\left|m_{a, i}-m_{b, j}\right| / 2} \\
& \times \frac{\left(x^{\left|m_{a, i}-m_{b, j}\right|+2-R_{a b}} z_{a, i} / z_{b, j} ; x^{2}\right)_{\infty}}{\left(x^{\left|m_{a, i}-m_{b, j}\right|+R_{a b}} z_{b, j} / z_{a, i} ; x^{2}\right)_{\infty}} .
\end{aligned}
$$

The bifundamental factor $\mathcal{I}_{\mathrm{B}}$ satisfies the identity

$$
\mathcal{I}_{\mathrm{B}}\left(\left(z_{a}, m_{a}\right),\left(z_{b}, m_{b}\right) ; R_{a b}\right) \mathcal{I}_{\mathrm{B}}\left(\left(z_{b}, m_{b}\right),\left(z_{a}, m_{a}\right) ; R_{b a}\right)=1
$$

for $R_{a b}+R_{b a}=2$.

We can connect the supersymmetric index to a lattice model by following the same procedure as before. We define an IRF model on the lattice drawn on the 46-torus by the NS5-branes. The state variables are $\left(z_{a}, m_{a}\right)$, and the Bolzmann weight is given by the same picture (3.27). By construction, the supersymmetric index of a $3 \mathrm{~d}$ brane box model coincides with the partition function of this IRF model. The Yang-Baxter equation again boils down to the equality (3.31) between the supersymmetric indices of two gauge theories. This equality, if true, establishes the integrability of the IRF model.

The equality indeed follows from the $\mathrm{SU}(N)$ version $[76,77]$ of the duality for $3 \mathrm{~d}$ $\mathcal{N}=2$ gauge theories proposed by Aharony [19]. There is an interesting twist, though: the theories described by the quivers that appear in the equality are not dual to each other.

The correct duality takes the theory on the left-hand side to a theory whose matter content consists of the chiral multiplets described by the quiver on the right-hand side, plus two extra singlets $V_{+}, V_{-}$. More generally, Aharony duality relates two $3 \mathrm{~d} \mathcal{N}=2$ gauge theories with the following matter contents:

\begin{tabular}{|l|cccccc|}
\hline & $G$ & $\mathrm{SU}\left(N_{f}\right)_{L}$ & $\mathrm{SU}\left(N_{f}\right)_{R}$ & $\mathrm{U}(1)_{B}$ & $\mathrm{U}(1)_{A}$ & $\mathrm{U}(1)_{R}$ \\
\hline$Q$ & $\square$ & $\bar{\square}$ & $\mathbf{1}$ & 1 & 1 & $1-N_{c} / N_{f}$ \\
$\widetilde{Q}$ & $\bar{\square}$ & $\mathbf{1}$ & $\square$ & -1 & 1 & $1-N_{c} / N_{f}$ \\
\hdashline$q$ & $\square$ & $\bar{\square}$ & $\mathbf{1}$ & $N_{c} /\left(N_{f}-N_{c}\right)$ & -1 & $N_{c} / N_{f}$ \\
$\tilde{q}$ & $\bar{q}$ & $\mathbf{1}$ & $\square$ & $-N_{c} /\left(N_{f}-N_{c}\right)$ & -1 & $N_{c} / N_{f}$ \\
$M$ & $\mathbf{1}$ & $\square$ & $\bar{\square}$ & 0 & 2 & $2\left(1-N_{c} / N_{f}\right)$ \\
$V_{ \pm}$ & $\mathbf{1}$ & $\mathbf{1}$ & $\mathbf{1}$ & 0 & $-N_{f}$ & 1 \\
\hline
\end{tabular}


The gauge group $G$ is $\mathrm{SU}\left(N_{c}\right)$ for the theory with $Q, \widetilde{Q}$, and $\mathrm{SU}\left(N_{f}-N_{c}\right)$ for its dual. Although a proof has not been given yet, there is strong evidence for the equality of the supersymmetric indices of these two theories in terms of series expansion in $x$ [77-79]. ${ }^{11}$

The above table contains one more global symmetry compared to its $4 \mathrm{~d}$ counterpart (3.32), namely the axial symmetry $\mathrm{U}(1)_{A}$ which is anomalous in four dimensions. In the definition of the index (5.2), we did not include fugacities for axial symmetries. Thanks to this omission, $V_{ \pm}$always have $R=1$ for whatever shift of $R$ by the relevant $\mathrm{U}(1)$ flavor symmetries, and their contributions to the index cancel since we can turn on a superpotential $W=m V_{+} V_{-}$. If we included these fugacities, the cancellation would no longer occur. In that case, the presence of $V_{ \pm}$would be crucial for the indices of the two theories to match.

Why does the logic fail if the fugacities for axial symmetries are turned on? The point is that when we apply T-duality to get a $3 \mathrm{~d}$ brane box model from a $4 \mathrm{~d}$ one, the 2-direction is compactified to a circle, however small its radius may be. In other words, we are really compactifying the $4 \mathrm{~d}$ theory on $S^{1}$ with finite radius, not dimensional reducing it. As emphasized in [76], the effective $3 \mathrm{~d}$ theory obtained by compactification has a superpotential that contains monopole operators. This superpotential breaks the axial symmetries, thereby reproducing the effect of anomalies in the $4 \mathrm{~d}$ theory. So it is not that the logic fails. Rather, we have no fugacities to turn on to begin with. Upon inclusion of such superpotentials on both sides, the extra multiplets disappear from the duality.

The axial symmetries are recovered if we decompactify the 2-direction. Although it is perfectly fine to do so, the resulting system is not related to a $4 \mathrm{~d}$ brane box model by Tduality and its connection to a lattice model requires a more careful analysis. It is not clear whether these additional symmetries admit a natural interpretation in the lattice model.

\subsection{2d brane box models}

Applying further T-duality along the 1-direction, we obtain the following brane configuration realizing a $2 \mathrm{~d} \mathcal{N}=(2,2)$ quiver gauge theory:

\begin{tabular}{|l|cccccccccc|}
\hline & 0 & 1 & 2 & 3 & 4 & 5 & 6 & 7 & 8 & 9 \\
\hline D3 & $\times$ & & & $\times$ & $\times$ & & $\times$ & & & \\
NS5 & $\times$ & $\times$ & $\times$ & $\times$ & $\times$ & $\times$ & & & & \\
NS5 & $\times$ & $\times$ & $\times$ & $\times$ & & & $\times$ & $\times$ & & \\
\hline
\end{tabular}

This theory is described by the same quiver diagram as in the $3 \mathrm{~d}$ and $4 \mathrm{~d}$ brane box cases. However, each node now represents a $\mathrm{U}(N)$ gauge group, not $\mathrm{SU}(N)$. The reason is that unlike the case with D4- or D5-branes, motion of D3-brane segments along NS5-branes creates disturbances on the NS5-branes that fall off sufficiently rapidly, and costs only a finite amount of energy. (In the $3 \mathrm{~d}$ brane box case, on the other hand, the required energy becomes arbitrarily large as we increase the radius of the 2-circle.)

\footnotetext{
${ }^{11}$ For $N_{c}=2$ and $N_{f}=3,4$, the equality was recently proved in [80] in the absence of the fugacity for $\mathrm{U}(1)_{A}$.
} 
The supersymmetric index of a $2 \mathrm{~d} \mathcal{N}=(2,2)$ theory is known as the elliptic genus. It is given by

$$
\mathcal{I}\left(q, y ;\left\{r_{\alpha}\right\}\right)=\operatorname{Tr}_{S^{1}}\left((-1)^{F} q^{H_{L}} y^{R / 2}\right),
$$

where $H_{L}$ is the left-moving Hamiltonian, and $R$ is defined as twice the left-moving $\mathrm{R}$ charge so that a superpotential preserving the R-symmetry has $R=2$. The trace receives contributions only from states with the right-moving Hamiltonian $H_{R}=0$. The elliptic genus can be computed as the partition function with twisted boundary conditions on a torus whose complex parameter $\tau$ is given by $q=e^{2 \pi \tau}$. As such, it enjoys a nice modular property.

An integral formula for the elliptic genus of an $\mathcal{N}=(2,2)$ gauge theory was derived in $[81-83]$. For the theory we are considering, the formula reads ${ }^{12}$

$$
\mathcal{I}=\oint \prod_{@} \frac{1}{N !} \prod_{j=1}^{N} \frac{\mathrm{d} u_{a, j}}{2 \pi i} \mathcal{I}_{\mathrm{V}}\left(u_{a}\right) \prod_{(a) \rightarrow(b)} \mathcal{I}_{\mathrm{B}}\left(u_{a}, u_{b} ; R_{a b}\right),
$$

with the vector and bifundamental multplet factors given by

$$
\begin{aligned}
\mathcal{I}_{\mathrm{V}}\left(u_{a}\right) & =\left(\frac{2 \pi \eta(\tau)^{3}}{\theta_{1}(-z \mid \tau)}\right)^{N} \prod_{i \neq j} \frac{\theta_{1}\left(u_{a, i}-u_{a, j} \mid \tau\right)}{\theta_{1}\left(u_{a, i}-u_{a, j}-z \mid \tau\right)}, \\
\mathcal{I}_{\mathrm{B}}\left(u_{a}, u_{b} ; R_{a b}\right) & =\prod_{i, j} \frac{\theta_{1}\left(u_{b, j}-u_{a, i}+\left(R_{a b} / 2-1\right) z \mid \tau\right)}{\theta_{1}\left(u_{b, j}-u_{a, i}+R_{a b} z / 2 \mid \tau\right)} .
\end{aligned}
$$

Here $z$ is related to the fugacity $y$ by $y=e^{2 \pi i z}$, and

$$
\begin{aligned}
\eta(\tau) & =q^{1 / 24} \prod_{n=1}^{\infty}\left(1-q^{n}\right), \\
\theta_{1}(z \mid \tau) & =-i \sum_{n=-\infty}^{\infty}(-1)^{n} e^{2 \pi i z(n+1 / 2)} e^{\pi i \tau(n+1 / 2)^{2}}
\end{aligned}
$$

are the Dedekind eta function and a Jacobi theta function. Note that $\theta_{1}(-z \mid \tau)=-\theta_{1}(z \mid \tau)$. As a consequence, $\mathcal{I}_{\mathrm{B}}$ obeys the identity (3.28).

The above formula takes the same form as the $4 \mathrm{~d}$ index formula (3.24) under the identification $z_{a, i}=e^{2 \pi i u_{a, i}}$. The subtlety lies in the integration contour, as was elucidated in $[82,83]$ with a careful path integral analysis. Since $\theta_{1}(z \mid \tau)$ has a pole at $z=0$, the integrand has poles at various places in the $u$-space, and the contour must pick up the residues at some but not all of these poles. There are many choices that lead to the correct answer. For us, a simple choice is such that for each node $a$, every $u_{a, i}$ encircles the pole located at $u_{a, i}=u_{b, j}-R_{b a} z / 2$ for some $(b, j)$, which comes from the factor $\mathcal{I}_{\mathrm{B}}\left(u_{b}, u_{a} ; R_{b a}\right)$ associated to the incoming arrow

$$
\text { (a) (b) . }
$$

\footnotetext{
${ }^{12}$ For concreteness we will follow the treatment in $[82,83]$ where the elliptic genus is defined in the $(R, R)$ sector, though the considerations given below equally apply to the other sectors. See [81] for the formula for the (NS, NS) sector.
} 
Moreover, $u_{a, i}$ and $u_{a, j}$ for $i \neq j$ must encircle different poles. We sum over the contributions from all such contours. Alternatively, we may choose the contours to encircle poles coming from outgoing arrows. The two choices give the same result, up to an overall sign. We refer the reader to $[82,83]$ for more details on the integration contour in general, and section 4.6 of [83] for details specific to theories closely related to ours.

As in the higher-dimensional cases, the supersymmetric index of a $2 \mathrm{~d}$ brane box model coincides with the partition function of an IRF model. The Boltzmann weight is given by the formula (3.27), and the Yang-Baxter equation reduces to the equality (3.31). This time, let us demonstrate this equality explicitly.

The elliptic genus of the theory described by the quiver on the left-hand side is computed by the contour integral

$$
\begin{aligned}
\mathcal{I}_{\mathrm{LHS}}=\frac{1}{N !} \oint \prod_{j=1}^{N} \frac{\mathrm{d} u_{g, j}}{2 \pi i} \mathcal{I}_{\mathrm{V}}\left(u_{g}\right) \mathcal{I}_{\mathrm{B}}\left(u_{a}, u_{g} ; R_{a g}\right) \mathcal{I}_{\mathrm{B}}\left(u_{d}, u_{g} ; R_{d g}\right) \\
\\
\quad \times \mathcal{I}_{\mathrm{B}}\left(u_{g}, u_{c} ; R_{g c}\right) \mathcal{I}_{\mathrm{B}}\left(u_{g}, u_{f} ; R_{g f}\right) .
\end{aligned}
$$

We choose to evaluate it with contours that encircle $N$ poles from the factors associated with the incoming arrows. For instance, suppose that we are evaluating the integral for contours such that the unordered set of these poles is $\left\{u_{a, j}-R_{a g} z / 2\right\}$, that is, each $u_{g, i^{-}}$ integral picks up the residue at $u_{g, i}=u_{a, j}-R_{a g} z / 2$ for some $j$. The $N$ ! different ways to assign the poles to the $u_{g, i}$ make equal contributions, so we only need to consider the case when $u_{g, i}=u_{a, i}-R_{a g} z / 2$ for all $i$. Using the fact that the residue of $1 / \theta_{1}(u \mid \tau)$ at $u=0$ is $1 / 2 \pi \eta(\tau)^{3}$, we find that the sum of the contributions from these contours is

$$
\mathcal{I}_{\mathrm{B}}\left(u_{a}, u_{c} ; R_{a g}+R_{g c}\right) \mathcal{I}_{\mathrm{B}}\left(u_{a}, u_{f} ; R_{a g}+R_{g f}\right) \mathcal{I}_{\mathrm{B}}\left(u_{d}, u_{a} ; R_{d g}-R_{a g}\right) .
$$

On the other hand, the elliptic genus of the right-hand side is given by

$$
\begin{aligned}
\mathcal{I}_{\mathrm{RHS}}=\frac{1}{N !} \oint \prod_{j=1}^{N} \frac{\mathrm{d} u_{g, j}}{2 \pi i} & \mathcal{I}_{\mathrm{V}}\left(u_{g}\right) \mathcal{I}_{\mathrm{B}}\left(u_{g}, u_{d} ; R_{g d}\right) \mathcal{I}_{\mathrm{B}}\left(u_{g}, u_{a} ; R_{g a}\right) \\
& \times \mathcal{I}_{\mathrm{B}}\left(u_{c}, u_{g} ; R_{c g}\right) \mathcal{I}_{\mathrm{B}}\left(u_{f}, u_{g} ; R_{f g}\right) \\
& \times \mathcal{I}_{\mathrm{B}}\left(u_{a}, u_{c} ; R_{a c}\right) \mathcal{I}_{\mathrm{B}}\left(u_{a}, u_{f} ; R_{a f}\right) \mathcal{I}_{\mathrm{B}}\left(u_{d}, u_{c} ; R_{d c}\right) \mathcal{I}_{\mathrm{B}}\left(u_{d}, u_{f} ; R_{d f}\right) .
\end{aligned}
$$

For the dual theory, we choose the poles from the outgoing arrows. The contours picking up the residues at the poles $\left\{u_{d, i}+R_{g d} / 2\right\}$ contribute

$$
\begin{aligned}
\mathcal{I}_{\mathrm{B}}\left(u_{d}, u_{a} ; R_{g a}-\right. & \left.R_{g d}\right) \mathcal{I}_{\mathrm{B}}\left(u_{c}, u_{d} ; R_{c g}+R_{g d}\right) \mathcal{I}_{\mathrm{B}}\left(u_{f}, u_{d} ; R_{f g}+R_{g d}\right) \\
& \times \mathcal{I}_{\mathrm{B}}\left(u_{a}, u_{c} ; R_{a c}\right) \mathcal{I}_{\mathrm{B}}\left(u_{a}, u_{f} ; R_{a f}\right) \mathcal{I}_{\mathrm{B}}\left(u_{d}, u_{c} ; R_{d c}\right) \mathcal{I}_{\mathrm{B}}\left(u_{d}, u_{f} ; R_{d f}\right) .
\end{aligned}
$$

After canceling out some factors using the identity (3.28), we see that this is equal to the contribution (5.17) found above.

In a similar fashion, one can show that for each set of poles $\left\{\hat{u}_{g, i}\right\} \subset\left\{u_{a, i}-R_{a g} z / 2\right\} \cup$ $\left\{u_{d, i}-R_{d g} z / 2\right\}$ in the theory on the left-hand side, there is a set of poles $\left\{\bar{u}_{g, i}\right\} \subset\left\{u_{a, i}+\right.$ $\left.R_{g a} z / 2\right\} \cup\left\{u_{d, i}+R_{g d} z / 2\right\}$ in the theory on the right-hand side such that the contours 
encircling these poles give the same contributions to the elliptic genera of the respective theories. (In the case that the R-charges vanish, $\left\{\bar{u}_{g, i}\right\}$ is the complement of $\left\{\hat{u}_{g, i}\right\}$ in $\left\{u_{a, i}\right\} \cup\left\{u_{d, i}\right\}$.) This is a one-to-one correspondence between the choices of poles in the two theories. Therefore, $\mathcal{I}_{\text {LHS }}=\mathcal{I}_{\text {RHS }}$.

The equality just proved is basically a consequence of a variant of Hori-Tong duality [20] proposed in [84]. However, it is important that the fugacities $u_{a}, u_{c}, u_{d}, u_{f}$ are unconstrained here. By contrast, when regarded as the elliptic genera of dual theories, the two sides of the equality must be evaluated with the constraint $\sum_{i}\left(u_{a, i}+u_{c, i}+u_{d, i}+u_{f, i}\right)=0$ since the diagonal $\mathrm{U}(1)$ subgroup of the flavor group $\mathrm{U}(N)_{a} \times \mathrm{U}(N)_{c} \times \mathrm{U}(N)_{d} \times \mathrm{U}(N)_{f}$ is gauged. So strictly speaking, the duality does not imply the Yang-Baxer equation. Also, note that the fugacity for the axial symmetry $\mathrm{U}(1)_{A}$ is set to zero as in the $3 \mathrm{~d}$ case. In general, this can be turned on in the elliptic genus.

Finally, we remark that the duality does not work if we replace the gauge group with $\mathrm{SU}(N)$. This is unlike the higher-dimensional cases, where one can gauge $\mathrm{U}(1)_{B}$ to obtain the duality for $\mathrm{U}(N)$ theories from that for $\mathrm{SU}(N)$ theories. Had we started with the dimensional reduction of the $3 \mathrm{~d}$ brane box model and simply discarded the $\mathrm{U}(1)_{A}$ fugacity, we would have reached a wrong conclusion.

\section{$62 \mathrm{~d} \mathcal{N}=(0,2)$ quiver gauge theories}

Looking at how the duality relation (3.31) was verified for $2 \mathrm{~d}$ brane box models, we notice that the numerator and denominator of the bifundamental factor $\mathcal{I}_{\mathrm{B}}$ played rather different roles. More specifically, while the denominator determined the pole structure and hence the possible choices for integration contours, the numerator only provided cancellations of some factors. The separation in their roles hints at the existence of integrability in less supersymmetric situations where an $\mathcal{N}=(2,2)$ chiral multiplet decomposes into two multiplets that correspond to the numerator and denominator.

$\mathcal{N}=(0,2)$ supersymmetry provides precisely the required decomposition: an $\mathcal{N}=$ $(2,2)$ chiral multiplet consists of an $\mathcal{N}=(0,2)$ chiral multiplet and Fermi multiplet, with the former corresponding to the denominator and the latter to the numerator. In this section we introduce three classes of $\mathcal{N}=(0,2)$ quiver gauge theories whose elliptic genera are captured by integrable lattice models. The first two classes lead to IRF models much like $\mathcal{N}=(2,2)$ brane box and brane tiling models discussed in section 5.2. The third class gives rise to an IRC model on $3 \mathrm{~d}$ lattices.

\section{1 $\mathcal{N}=(0,2)$ theories related to brane box configurations}

Let us take a quiver from some brane box model, and replace the vertical arrows with dotted arrows. (Of course, one may as well choose to replace the horizontal arrows instead.) For example, starting from the quiver for the $2 \times 3$ brane box model shown in figure 2 (b), we obtain the diagram in figure 13 . We interpret the resulting diagram as a quiver of an $\mathcal{N}=(0,2)$ gauge theory, letting dotted arrows represent bifundamental Fermi multiplets. As usual, circle nodes and solid arrows represent vector multiplets and bifundamental chiral 


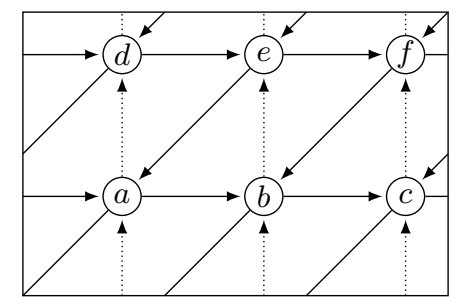

Figure 13. The $\mathcal{N}=(0,2)$ theory obtained from the $2 \times 3$ brane box model.

multiplets. By this procedure we can associate an $\mathcal{N}=(0,2)$ quiver gauge theory to every brane box model. The reader is referred to $[21,85]$ for basics of $\mathcal{N}=(0,2)$ gauge theories.

Although the left-moving R-symmetry is no longer present, the theory still has the flavor symmetries $\mathrm{U}(1)_{\alpha}$, at least classically. So we define the "R-charge" in the present case by

$$
R=\sum_{\alpha} r_{\alpha} F_{\alpha}
$$

Our claim is that the elliptic genus (5.9) of this theory is equal to the partition function of an integrable IRF model.

Before we give a proof of this claim, cautionary remarks are in order. As it is, the theory defined above suffers from mixed anomalies for the diagonal $U(1)$ factors of the $\mathrm{U}(N)$ gauge groups. Similarly, the flavor symmetry generated by $R$ is anomalous. These anomalies must be canceled by introduction of extra multiplets. For the moment let us ignore these issues; we will address them later.

The relevant formula for elliptic genera was derived in [82, 83]. The only difference compared to the $\mathcal{N}=(2,2)$ case is that it involves three kinds of factors, corresponding to the three kinds of multiplets:

$$
\mathcal{I}=\oint \prod_{(a)} \frac{1}{N !} \prod_{j=1}^{N} \frac{\mathrm{d} u_{a, j}}{2 \pi i} \mathcal{I}_{\mathrm{V}}\left(u_{a}\right) \prod_{(a) \rightarrow \text { (b) }} \mathcal{I}_{\mathrm{BC}}\left(u_{a}, u_{b} ; R_{a b}\right) \prod_{\text {(a) } \rightarrow \text { (b) }} \mathcal{I}_{\mathrm{BF}}\left(u_{a}, u_{b} ; R_{a b}\right),
$$

where the vector, bifundamental chiral and bifundamental Fermi multiplet factors are ${ }^{13}$

$$
\begin{aligned}
\mathcal{I}_{\mathrm{V}}\left(u_{a}\right) & =\left(\frac{2 \pi \eta(\tau)^{2}}{i}\right)^{N} \prod_{i \neq j} \frac{i \theta_{1}\left(u_{a, i}-u_{a, j} \mid \tau\right)}{\eta(\tau)}, \\
\mathcal{I}_{\mathrm{BC}}\left(u_{a}, u_{b} ; R_{a b}\right) & =\prod_{i, j} \frac{i \eta(\tau)}{\theta_{1}\left(u_{b, j}-u_{a, i}+R_{a b} z / 2 \mid \tau\right)}, \\
\mathcal{I}_{\mathrm{BF}}\left(u_{a}, u_{b} ; R_{a b}\right) & =\prod_{i, j} \frac{i \theta_{1}\left(u_{b, j}-u_{a, i}+R_{a b} z / 2 \mid \tau\right)}{\eta(\tau)} .
\end{aligned}
$$

Up to an immaterial overall sign, $\mathcal{I}_{\mathrm{BC}}\left(u_{a}, u_{b} ; R_{a b}\right) \mathcal{I}_{\mathrm{BF}}\left(u_{a}, u_{b} ; R_{a b}-2\right)$ is equal to the $\mathcal{N}=$ $(2,2)$ bifundamental chiral multiplet factor (5.12). Likewise, $\mathcal{I}_{\mathrm{V}}\left(u_{a}\right) \mathcal{I}_{\mathrm{BC}}\left(u_{a}, u_{a} ;-2\right)$ is equal to the $\mathcal{N}=(2,2)$ vector multiplet factor $(5.11)$, as is consistent with the decomposition

\footnotetext{
${ }^{13}$ See $[21,86]$ for the formulas for the (NS, NS) sector.
} 
of an $\mathcal{N}=(2,2)$ vector multiplet into an $\mathcal{N}=(0,2)$ vector multiplet and adjoint chiral multiplet. We have

$$
\mathcal{I}_{\mathrm{BC}}\left(u_{a}, u_{b} ; R_{a b}\right) \mathcal{I}_{\mathrm{BF}}\left(u_{b}, u_{a} ; R_{b a}\right)=1
$$

for $R_{a b}+R_{b a}=0$, or more graphically,

$$
a \sqrt{a}=a \quad b
$$

The cancellation of arrows corresponds to giving masses to a pair of chiral multiplet and Fermi multiplet.

For the theory under consideration, the elliptic genus can be computed as the partition function of an IRF model with Boltzmann weight

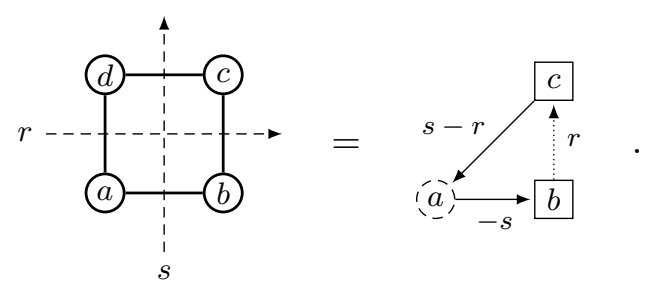

Plugging this into the Yang-Baxter equation, we find that the integrability of the model is equivalent to the equality

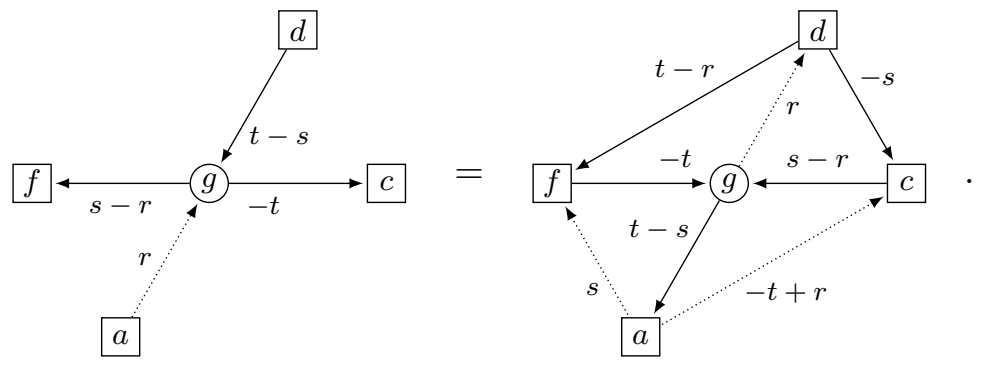

Let us demonstrate it.

The left-hand side is given by the contour integral

$$
\begin{aligned}
\mathcal{I}_{\mathrm{LHS}}=\frac{1}{N !} \oint \prod_{j=1}^{N} \frac{\mathrm{d} u_{g, j}}{2 \pi i} \mathcal{I}_{\mathrm{V}}\left(u_{g}\right) \mathcal{I}_{\mathrm{BC}}\left(u_{d}, u_{g} ; R_{d g}\right) & \mathcal{I}_{\mathrm{BF}}\left(u_{a}, u_{g} ; R_{a g}\right) \\
& \times \mathcal{I}_{\mathrm{BC}}\left(u_{g}, u_{c} ; R_{g c}\right) \mathcal{I}_{\mathrm{BC}}\left(u_{g}, u_{f} ; R_{g f}\right) .
\end{aligned}
$$

If we choose the contours to pick up residues from the incoming arrow, then the set of relevant poles is $\left\{u_{d, i}-R_{d g} z / 2\right\}$. Thus we have

$$
\mathcal{I}_{\mathrm{LHS}}=\mathcal{I}_{\mathrm{BF}}\left(u_{a}, u_{d} ; R_{a g}-R_{d g}\right) \mathcal{I}_{\mathrm{BC}}\left(u_{d}, u_{c} ; R_{d g}+R_{g c}\right) \mathcal{I}_{\mathrm{BC}}\left(u_{d}, u_{f} ; R_{d g}+R_{g f}\right) .
$$

The right-hand side of the equality (6.9) is

$$
\begin{aligned}
\mathcal{I}_{\mathrm{RHS}}=\frac{1}{N !} \oint \prod_{j=1}^{N} \frac{\mathrm{d}}{2} u_{g, j} & \mathcal{I}_{\mathrm{V}}\left(u_{g}\right) \mathcal{I}_{\mathrm{BC}}\left(u_{g}, u_{a} ; R_{g a}\right) \mathcal{I}_{\mathrm{BF}}\left(u_{g}, u_{d} ; R_{g d}\right) \\
& \times \mathcal{I}_{\mathrm{BC}}\left(u_{c}, u_{g} ; R_{c g}\right) \mathcal{I}_{\mathrm{BC}}\left(u_{f}, u_{g} ; R_{f g}\right) \\
& \times \mathcal{I}_{\mathrm{BF}}\left(u_{a}, u_{c} ; R_{a c}\right) \mathcal{I}_{\mathrm{BF}}\left(u_{a}, u_{f} ; R_{a f}\right) \mathcal{I}_{\mathrm{BC}}\left(u_{d}, u_{c} ; R_{d c}\right) \mathcal{I}_{\mathrm{BC}}\left(u_{d}, u_{f} ; R_{d f}\right)
\end{aligned}
$$




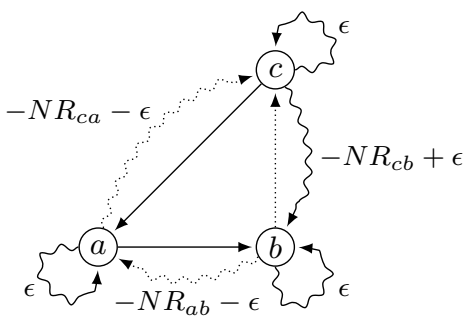

Figure 14. The R-charges of the extra multiplets for anomaly cancellation.

The "complement" set of poles is $\left\{u_{a, i}+R_{g a} z / 2\right\}$, and they give

$$
\begin{aligned}
\mathcal{I}_{\mathrm{RHS}}=\mathcal{I}_{\mathrm{BF}}\left(u_{a}, u_{d} ; R_{g d}-R_{g a}\right) \mathcal{I}_{\mathrm{BC}}\left(u_{c}, u_{a} ; R_{c g}+R_{g a}\right) \mathcal{I}_{\mathrm{BC}}\left(u_{f}, u_{a} ; R_{f g}+R_{g a}\right) \\
\quad \times \mathcal{I}_{\mathrm{BF}}\left(u_{a}, u_{c} ; R_{a c}\right) \mathcal{I}_{\mathrm{BF}}\left(u_{a}, u_{f} ; R_{a f}\right) \mathcal{I}_{\mathrm{BC}}\left(u_{d}, u_{c} ; R_{d c}\right) \mathcal{I}_{\mathrm{BC}}\left(u_{d}, u_{f} ; R_{d f}\right) .
\end{aligned}
$$

Canceling some factors using the identity (6.6), we find $\mathcal{I}_{\text {LHS }}=\mathcal{I}_{\text {RHS }}$.

In fact, the quivers on the two sides of the equality (6.9) describe two theories related by the $(0,2)$ triality transformation [21]. The third theory in the triality is the free theory with quiver

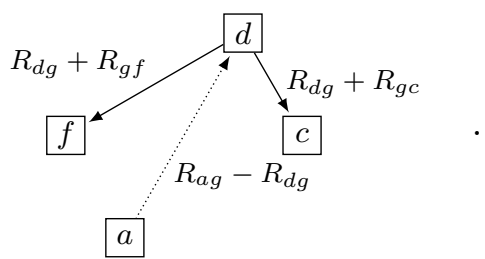

This is manifest in the expression (6.11) of the elliptic genus.

Finally, we come back to the issues of anomalies. To cancel the mixed U(1) gauge anomalies and $R$-anomaly, we add to each solid arrow

$$
(a) \rightarrow(b)
$$

a Fermi multiplet with $R=-N R_{a b}-\epsilon$ in the representation $\operatorname{det}_{a} \otimes \operatorname{det}_{b}^{-1}$ of $\mathrm{U}(N)_{a} \times \mathrm{U}(N)_{b}$, and to each dotted arrow

$$
\text { (a) - b }
$$

a chiral multiplet with $R=-N R_{a b}+\epsilon$ in $\operatorname{det}_{a} \otimes \operatorname{det}_{b}^{-1}$. Here $\epsilon$ is a nonzero parameter. Furthermore, to each node we introduce a singlet chiral multiplet with $R=\epsilon$, though this is not required for anomaly cancellation. We can visualize these extra multiplets as wavy arrows, as in figure 14 .

The presence of the extra multiplets modifies the factors associated to vector and bifundamental multiplets to

$$
\begin{aligned}
\mathcal{I}_{\mathrm{V}}^{\prime}\left(u_{a}\right) & =\frac{i \eta(\tau)}{\theta_{1}(\epsilon z / 2 \mid \tau)} \mathcal{I}_{\mathrm{V}}\left(u_{a}\right), \\
\mathcal{I}_{\mathrm{BC}}^{\prime}\left(u_{a}, u_{b} ; R_{a b}\right) & =\frac{i \theta_{1}\left(\sum_{i} u_{a, i}-\sum_{i} u_{b, i}+\left(-N R_{a b}-\epsilon\right) z / 2 \mid \tau\right)}{\eta(\tau)} \mathcal{I}_{\mathrm{BC}}\left(u_{a}, u_{b} ; R_{a b}\right), \\
\mathcal{I}_{\mathrm{BF}}^{\prime}\left(u_{a}, u_{b} ; R_{a b}\right) & =\frac{i \eta(\tau)}{\theta_{1}\left(\sum_{i} u_{a, i}-\sum_{i} u_{b, i}+\left(-N R_{a b}+\epsilon\right) z / 2 \mid \tau\right)} \mathcal{I}_{\mathrm{BF}}\left(u_{a}, u_{b} ; R_{a b}\right) .
\end{aligned}
$$




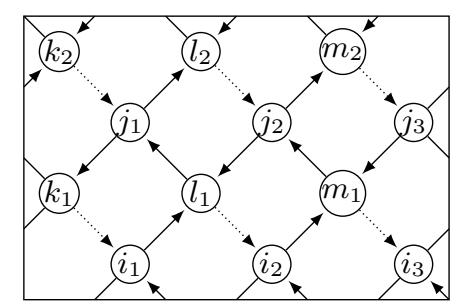

Figure 15. The $\mathcal{N}=(0,2)$ theory obtained from the $2 \times 3$ brane tiling model.

Apart from this modification, the computations of the elliptic genera for the equality (6.9) remain unchanged. In particular, we can use the same integration contours, since the extra multiplets introduce neither solid incoming arrows to the left-hand side nor solid outgoing arrows to the right-hand side.

\section{2 $\mathcal{N}=(0,2)$ theories related to brane tiling configurations}

Quivers from brane tiling models can also be modified to produce $\mathcal{N}=(0,2)$ theories that exhibit integrability. As in the brane box case discussed above, we obtain these theories by replacing one of the arrows in the R-matrix with a dotted arrow:

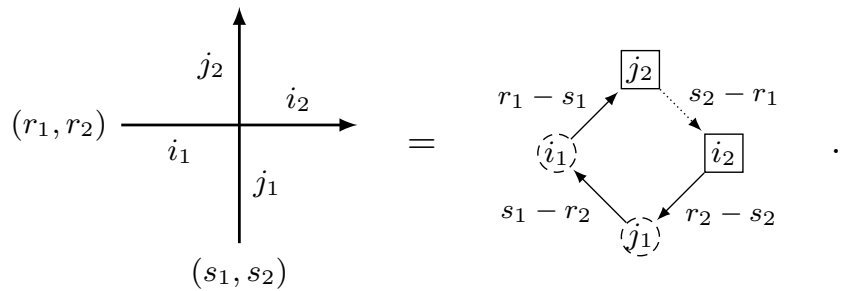

Extra multiplets for anomaly cancellations are implicit in this picture. Figure 15 shows the quiver for the $\mathcal{N}=(0,2)$ theory obtained from the $2 \times 3$ brane tiling model, whose quiver is given in figure 11(b).

The Yang-Baxter equation for this R-matrix reduces to the equality

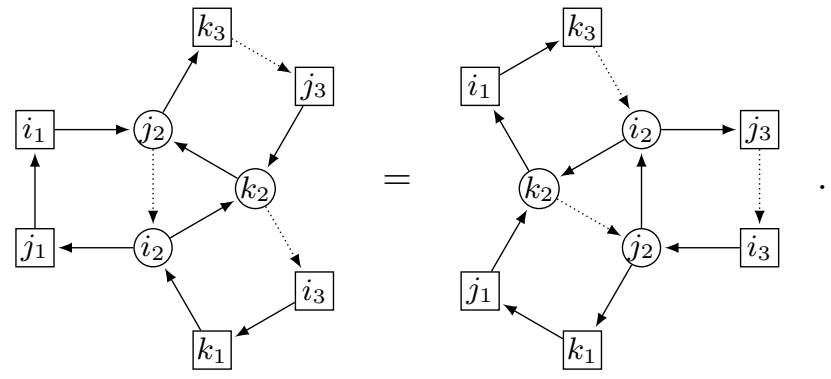

Application of the $(0,2)$ triality transformation (6.9) four times (more precisely, one triality transformation followed by three others in the opposite direction) turns the left-hand side into the right-hand side. This is illustrated in figure 16 .

\subsection{Brane cube models}

Lastly, we briefly discuss the class of $\mathcal{N}=(0,2)$ quiver gauge theories known as brane cube models [22]. These theories are constructed from brane configurations analogous to 

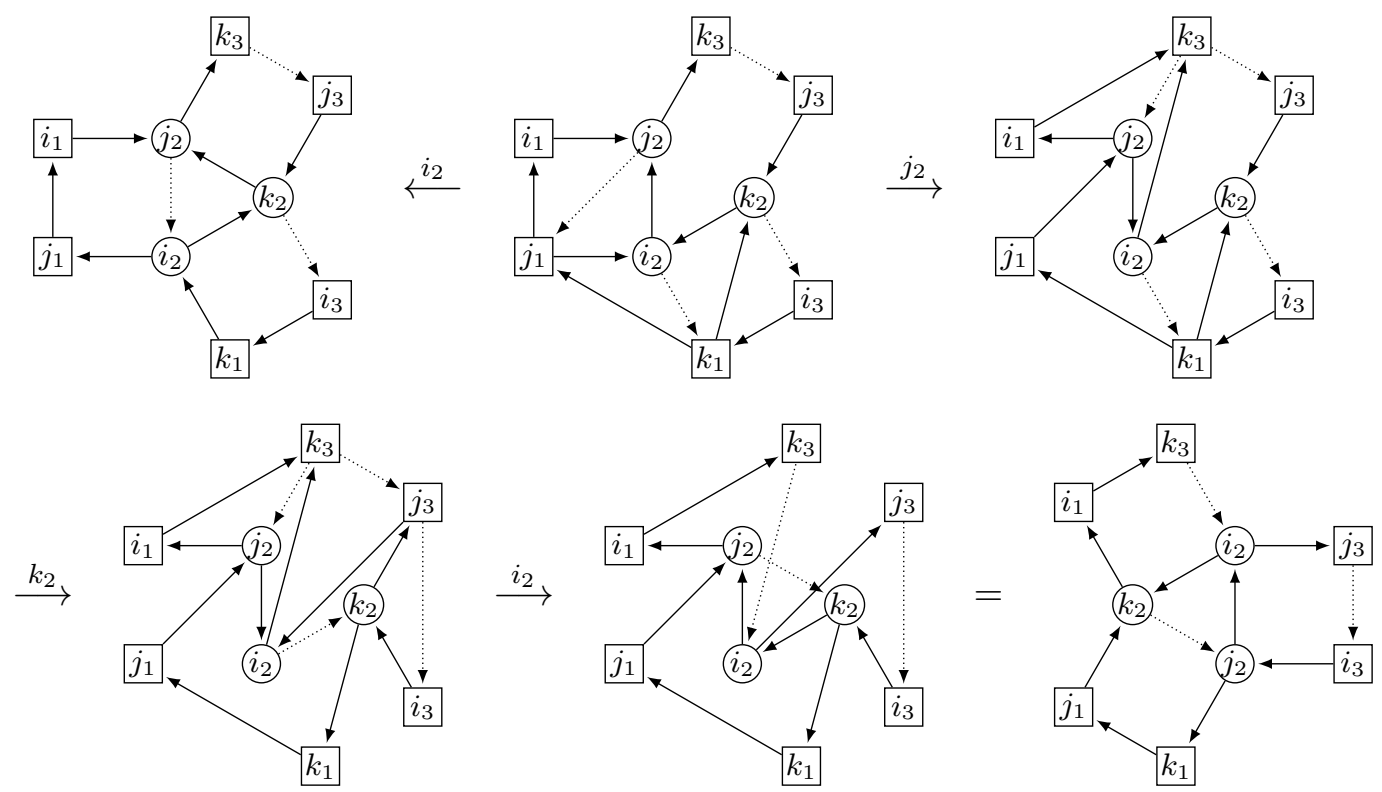

Figure 16. A sequence of $(0,2)$ triality transformations that relates the two sides of the YangBaxter equation (6.21). An arrow between two quivers represents a $(0,2)$ triality transformation in the direction from the right- to left-hand side of the equality (6.9).

brane boxes:

\begin{tabular}{|l|cccccccccc|}
\hline & 0 & 1 & 2 & 3 & 4 & 5 & 6 & 7 & 8 & 9 \\
\hline D4 & $\times$ & $\times$ & $\times$ & & $\times$ & & $\times$ & & & \\
NS5 & $\times$ & $\times$ & $\times$ & $\times$ & $\times$ & $\times$ & & & & \\
NS5 & $\times$ & $\times$ & $\times$ & $\times$ & & & $\times$ & $\times$ & & \\
NS5 & $\times$ & $\times$ & & & $\times$ & $\times$ & $\times$ & $\times$ & & \\
\hline
\end{tabular}

Note that brane cube models include $3 \mathrm{~d}$ brane box models (5.1) as special cases. A brane box model is characterized by the $2 \mathrm{~d}$ lattice drawn on the 46 -torus by the NS5-branes. For a brane cube model, the NS5-branes make a $3 \mathrm{~d}$ lattice in the 246 -space which we take to be a 3 -torus $T^{3}$.

A brane cube configuration can be thought of as describing intersecting codimension-1 defects in the $5 \mathrm{~d}$ theory on the D4-branes. We can topologically twist the theory along the 246 -space, replacing the holonomy group $\mathrm{SO}(3)_{246}$ with the diagonal subgroup of $\mathrm{SO}(3)_{246} \times$ $\mathrm{SO}(3)_{357}$. The twisted theory has four supercharges that are scalar in the 246-space, hence unbroken when this space is a general 3 -manifold $\Sigma$. These supercharges generate $\mathcal{N}=$ $(2,2)$ supersymmetry in the 01 -space. Half of them, generating an $\mathcal{N}=(0,2)$ subalgebra, are preserved by codimension- 1 defects supported on arbitrary surfaces inside $\Sigma$. Therefore, the twisted theory gives a morphism that sends a configuration of surfaces in $\Sigma$ to the effective $\mathcal{N}=(0,2)$ theory. Taking the elliptic genus, we get a $3 \mathrm{~d}$ TQFT on $\Sigma$ equipped with surface operators. An extra dimension emerges upon lifting the system to M-theory, and it follows that the elliptic genus of a brane cube model is equal to the partition function of a $3 \mathrm{~d}$ integrable lattice model.

Like an IRF model is associated with brane box models, the lattice model associated with brane cube models is an IRC model. According to the quiver rule found in [22], one 
way to describe this model is to set its Boltzmann weight

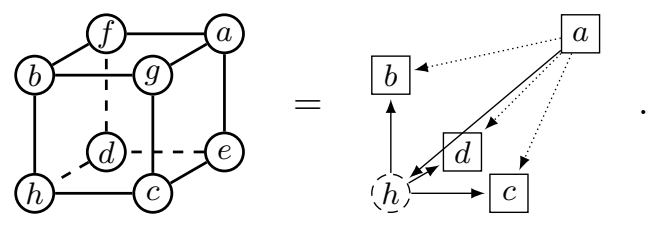

This Boltzmann weight, or another one that leads to the same IRC model on cubic lattices, should solve the tetrahedron equation (2.25). It would be interesting to verify this statement.

\section{Acknowledgments}

The author would like to thank Sergei Gukov and Edward Witten for helpful comments, and Nils Carqueville for valuable discussions. This work is supported by INFN Postdoctoral Fellowship.

Open Access. This article is distributed under the terms of the Creative Commons Attribution License (CC-BY 4.0), which permits any use, distribution and reproduction in any medium, provided the original author(s) and source are credited.

\section{References}

[1] V.V. Bazhanov and S.M. Sergeev, A master solution of the quantum Yang-Baxter equation and classical discrete integrable equations, Adv. Theor. Math. Phys. 16 (2012) 65 [arXiv: 1006.0651] [INSPIRE].

[2] V.V. Bazhanov and S.M. Sergeev, Elliptic gamma-function and multi-spin solutions of the Yang-Baxter equation, Nucl. Phys. B 856 (2012) 475 [arXiv:1106.5874] [INSPIRE].

[3] M. Kashiwara and T. Miwa, A class of elliptic solutions to the star triangle relation, Nucl. Phys. B 275 (1986) 121 [inSPIRE].

[4] G. von Gehlen and V. Rittenberg, $Z(n)$ symmetric quantum chains with an infinite set of conserved charges and $Z(n)$ zero modes, Nucl. Phys. B 257 (1985) 351 [INSPIRE].

[5] H. Au-Yang, B.M. McCoy, J.H.H. perk, S. Tang and M.-L. Yan, Commuting transfer matrices in the chiral Potts models: solutions of star triangle equations with genus $>1$, Phys. Lett. A 123 (1987) 219 [INSPIRE].

[6] R.J. Baxter, J.H.H. Perk and H. Au-Yang, New solutions of the star triangle relations for the chiral Potts model, Phys. Lett. A 128 (1988) 138 [INSPIRE].

[7] V.P. Spiridonov, Elliptic beta integrals and solvable models of statistical mechanics, Contemp. Math. 563 (2012) 181 [arXiv: 1011.3798] [INSPIRE].

[8] M. Yamazaki, Quivers, YBE and 3-manifolds, JHEP 05 (2012) 147 [arXiv:1203.5784] [INSPIRE].

[9] M. Yamazaki, New integrable models from the gauge/YBE correspondence, J. Statist. Phys. 154 (2014) 895 [arXiv: 1307.1128] [INSPIRE].

[10] S. Franco, A. Hanany, K.D. Kennaway, D. Vegh and B. Wecht, Brane dimers and quiver gauge theories, JHEP 01 (2006) 096 [hep-th/0504110] [INSPIRE]. 
[11] E. Witten, Solutions of four-dimensional field theories via M-theory, Nucl. Phys. B 500 (1997) 3 [hep-th/9703166] [INSPIRE].

[12] D. Gaiotto, $N=2$ dualities, JHEP 08 (2012) 034 [arXiv:0904.2715] [INSPIRE].

[13] D. Gaiotto, G.W. Moore and A. Neitzke, Wall-crossing, Hitchin systems and the WKB approximation, arXiv:0907.3987 [INSPIRE].

[14] A. Gadde, E. Pomoni, L. Rastelli and S.S. Razamat, S-duality and $2 D$ topological QFT, JHEP 03 (2010) 032 [arXiv:0910.2225] [INSPIRE].

[15] F. Benini, T. Nishioka and M. Yamazaki, $4 D$ index to $3 D$ index and $2 D$ TQFT, Phys. Rev. D 86 (2012) 065015 [arXiv:1109.0283] [INSPIRE].

[16] K. Costello, Integrable lattice models from four-dimensional field theories, Proc. Symp. Pure Math. 88 (2014) 3 [arXiv:1308.0370] [INSPIRE].

[17] K. Costello, Supersymmetric gauge theory and the Yangian, arXiv:1303.2632 [INSPIRE].

[18] A. Hanany and A. Zaffaroni, On the realization of chiral four-dimensional gauge theories using branes, JHEP 05 (1998) 001 [hep-th/9801134] [INSPIRE].

[19] O. Aharony, IR duality in $D=3 N=2$ supersymmetric $\operatorname{USp}\left(2 N_{c}\right)$ and $\mathrm{U}\left(N_{c}\right)$ gauge theories, Phys. Lett. B 404 (1997) 71 [hep-th/9703215] [INSPIRE].

[20] K. Hori and D. Tong, Aspects of non-abelian gauge dynamics in two-dimensional $N=(2,2)$ theories, JHEP 05 (2007) 079 [hep-th/0609032] [INSPIRE].

[21] A. Gadde, S. Gukov and P. Putrov, (0,2) trialities, JHEP 03 (2014) 076 [arXiv:1310.0818] [INSPIRE].

[22] H. Garcia-Compean and A.M. Uranga, Brane box realization of chiral gauge theories in two-dimensions, Nucl. Phys. B 539 (1999) 329 [hep-th/9806177] [INSPIRE].

[23] A.B. Zamolodchikov, Tetrahedra equations and integrable systems in three-dimensional space, Sov. Phys. JETP 52 (1980) 325.

[24] A.B. Zamolodchikov, Tetrahedron equations and the relativistic $S$ matrix of straight strings in (2+1)-dimensions, Commun. Math. Phys. 79 (1981) 489 [InSPIRE].

[25] A. Gadde and M. Yamazaki, $(0,2)$ trialities and the tetrahedron equation, to appear.

[26] D. Gaiotto, G.W. Moore and A. Neitzke, Four-dimensional wall-crossing via three-dimensional field theory, Commun. Math. Phys. 299 (2010) 163 [arXiv:0807.4723] [INSPIRE].

[27] S. Alexandrov and P. Roche, TBA for non-perturbative moduli spaces, JHEP 06 (2010) 066 [arXiv: 1003.3964] [INSPIRE].

[28] N.A. Nekrasov and S.L. Shatashvili, Supersymmetric vacua and Bethe ansatz, Nucl. Phys. Proc. Suppl. 192-193 (2009) 91 [arXiv:0901.4744] [INSPIRE].

[29] N.A. Nekrasov and S.L. Shatashvili, Quantum integrability and supersymmetric vacua, Prog. Theor. Phys. Suppl. 177 (2009) 105 [arXiv:0901.4748] [INSPIRE].

[30] N.A. Nekrasov and S.L. Shatashvili, Quantization of integrable systems and four dimensional gauge theories, arXiv:0908.4052 [INSPIRE].

[31] D. Sadri and M.M. Sheikh-Jabbari, Integrable spin chains on the conformal moose, JHEP 03 (2006) 024 [hep-th/0510189] [INSPIRE]. 
[32] E. Witten, Gauge theories and integrable lattice models, Nucl. Phys. B 322 (1989) 629 [INSPIRE].

[33] E. Witten, Gauge theories, vertex models and quantum groups, Nucl. Phys. B 330 (1990) 285 [INSPIRE].

[34] A. Kapustin, Holomorphic reduction of $N=2$ gauge theories, Wilson-'t Hooft operators and S-duality, hep-th/0612119 [INSPIRE].

[35] Y. Fukuda, T. Kawano and N. Matsumiya, 5D SYM and 2D q-deformed YM, Nucl. Phys. B 869 (2013) 493 [arXiv:1210.2855] [INSPIRE].

[36] T. Dimofte, S. Gukov and L. Hollands, Vortex counting and Lagrangian 3-manifolds, Lett. Math. Phys. 98 (2011) 225 [arXiv: 1006.0977] [INSPIRE].

[37] Y. Luo, M.-C. Tan, J. Yagi and Q. Zhao, $\Omega$-deformation of B-twisted gauge theories and the $3 D-3 D$ correspondence, JHEP 02 (2015) 047 [arXiv: 1410.1538] [INSPIRE].

[38] J. Yagi, 3D TQFT from 6D SCFT, JHEP 08 (2013) 017 [arXiv:1305.0291] [INSPIRE].

[39] S. Lee and M. Yamazaki, 3D Chern-Simons theory from M5-branes, JHEP 12 (2013) 035 [arXiv: 1305.2429] [INSPIRE].

[40] C. Cordova and D.L. Jafferis, Complex Chern-Simons from M5-branes on the squashed three-sphere, arXiv: 1305.2891 [INSPIRE].

[41] T. Dimofte, D. Gaiotto and S. Gukov, Gauge theories labelled by three-manifolds, Commun. Math. Phys. 325 (2014) 367 [arXiv:1108.4389] [INSPIRE].

[42] Y. Terashima and M. Yamazaki, $\mathrm{SL}(2, R)$ Chern-Simons, Liouville, and gauge theory on duality walls, JHEP 08 (2011) 135 [arXiv:1103.5748] [INSPIRE].

[43] T. Dimofte, Complex Chern-Simons theory at level $k$ via the $3 D-3 D$ correspondence, Commun. Math. Phys. 339 (2015) 619 [arXiv:1409.0857] [INSPIRE].

[44] J. Yagi, On the six-dimensional origin of the AGT correspondence, JHEP 02 (2012) 020 [arXiv:1112.0260] [INSPIRE].

[45] J. Yagi, Compactification on the $\Omega$-background and the AGT correspondence, JHEP 09 (2012) 101 [arXiv:1205.6820] [INSPIRE].

[46] A. Gadde, S. Gukov and P. Putrov, Fivebranes and 4-manifolds, arXiv:1306.4320 [INSPIRE].

[47] G.W. Moore and Y. Tachikawa, On 2D TQFTs whose values are holomorphic symplectic varieties, Proc. Symp. Pure Math. 85 (2012) 191 [arXiv:1106.5698] [InSPIRE].

[48] R.J. Baxter, The Yang-Baxter equations and the Zamolodchikov model, Phys. D 18 (1986) 321.

[49] A. Hanany and E. Witten, Type IIB superstrings, BPS monopoles and three-dimensional gauge dynamics, Nucl. Phys. B 492 (1997) 152 [hep-th/9611230] [INSPIRE].

[50] C. Romelsberger, Counting chiral primaries in $N=1, D=4$ superconformal field theories, Nucl. Phys. B 747 (2006) 329 [hep-th/0510060] [INSPIRE].

[51] J. Kinney, J.M. Maldacena, S. Minwalla and S. Raju, An index for 4 dimensional super conformal theories, Commun. Math. Phys. 275 (2007) 209 [hep-th/0510251] [INSPIRE].

[52] G. Festuccia and N. Seiberg, Rigid supersymmetric theories in curved superspace, JHEP 06 (2011) 114 [arXiv:1105.0689] [INSPIRE]. 
[53] D. Sen, Supersymmetry in the space-time $R \times S^{3}$, Nucl. Phys. B 284 (1987) 201 [InSPIRE].

[54] C. Closset, T.T. Dumitrescu, G. Festuccia and Z. Komargodski, The geometry of supersymmetric partition functions, JHEP 01 (2014) 124 [arXiv:1309.5876] [INSPIRE].

[55] E. Witten, A new look at the path integral of quantum mechanics, in Surveys in differential geometry. Volume XV. Perspectives in mathematics and physics, Inernational Press, Somerville U.S.A. (2011), arXiv: 1009.6032 [INSPIRE].

[56] E. Witten, Fivebranes and knots, Quantum Topol. 3 (2012) 1 [arXiv:1101.3216] [InSPIRE].

[57] C. Beem, T. Dimofte and S. Pasquetti, Holomorphic blocks in three dimensions, JHEP 12 (2014) 177 [arXiv:1211.1986] [INSPIRE].

[58] F.A. Dolan and H. Osborn, Applications of the superconformal index for protected operators and q-hypergeometric identities to $N=1$ dual theories, Nucl. Phys. B 818 (2009) 137 [arXiv: 0801.4947] [INSPIRE].

[59] E. M. Rains, Transformations of elliptic hypergeometric integrals, Ann. Math. 171 (2010) 169 [math/0309252].

[60] V.P. Spiridonov, On the elliptic beta function, Russ. Math. Surv. 56 (2001) 185.

[61] V. Spiridonov, Theta hypergeometric series, in Asymptotic combinatorics with application to mathematical physics, V. Malyshev and A. Vershik eds., NATO Science Series volume 77, Springer, Germany (2002).

[62] V. P. Spiridonov and S. O. Warnaar, Inversions of integral operators and elliptic beta integrals on root systems, Adv. Math. 207 (2006) 91 [math/0411044].

[63] S. Elitzur, A. Giveon and D. Kutasov, Branes and $N=1$ duality in string theory, Phys. Lett. B 400 (1997) 269 [hep-th/9702014] [INSPIRE].

[64] S. Elitzur, A. Giveon, D. Kutasov, E. Rabinovici and A. Schwimmer, Brane dynamics and $N=1$ supersymmetric gauge theory, Nucl. Phys. B 505 (1997) 202 [hep-th/9704104] [INSPIRE].

[65] Y. Imamura, Global symmetries and 't Hooft anomalies in brane tilings, JHEP 12 (2006) 041 [hep-th/0609163] [INSPIRE].

[66] K.D. Kennaway, Brane tilings, Int. J. Mod. Phys. A 22 (2007) 2977 [arXiv:0706.1660] [INSPIRE].

[67] M. Yamazaki, Brane tilings and their applications, Fortsch. Phys. 56 (2008) 555 [arXiv: 0803.4474] [INSPIRE].

[68] V.P. Spiridonov, A bailey tree for integrals, Theor. Math. Phys. 139 (2004) 536 [math/0312502].

[69] S.E. Derkachov and V.P. Spiridonov, Yang-Baxter equation, parameter permutations and the elliptic beta integral, Russ. Math. Surveys 68 (2013) 1027 [arXiv:1205.3520] [INSPIRE].

[70] V.P. Spiridonov, Aspects of elliptic hypergeometric functions, arXiv:1307.2876 [INSPIRE].

[71] A. Hanany and D. Vegh, Quivers, tilings, branes and rhombi, JHEP 10 (2007) 029 [hep-th/0511063] [INSPIRE].

[72] Y. Imamura and S. Yokoyama, Index for three dimensional superconformal field theories with general R-charge assignments, JHEP 04 (2011) 007 [arXiv: 1101.0557] [INSPIRE]. 
[73] C. Closset, T.T. Dumitrescu, G. Festuccia and Z. Komargodski, Supersymmetric field theories on three-manifolds, JHEP 05 (2013) 017 [arXiv:1212.3388] [INSPIRE].

[74] S. Kim, The complete superconformal index for $N=6$ Chern-Simons theory, Nucl. Phys. B 821 (2009) 241 [Erratum ibid. B 864 (2012) 884] [arXiv:0903.4172] [INSPIRE].

[75] A. Kapustin and B. Willett, Generalized superconformal index for three dimensional field theories, arXiv:1106.2484 [INSPIRE].

[76] O. Aharony, S.S. Razamat, N. Seiberg and B. Willett, $3 D$ dualities from $4 D$ dualities, JHEP 07 (2013) 149 [arXiv: 1305. 3924] [INSPIRE].

[77] J. Park and K.-J. Park, Seiberg-like dualities for $3 D N=2$ theories with $\mathrm{SU}(N)$ gauge group, JHEP 10 (2013) 198 [arXiv: 1305.6280] [INSPIRE].

[78] C. Hwang, H. Kim, K.-J. Park and J. Park, Index computation for 3D Chern-Simons matter theory: test of Seiberg-like duality, JHEP 09 (2011) 037 [arXiv:1107.4942] [INSPIRE].

[79] C. Hwang, H.-C. Kim and J. Park, Factorization of the 3D superconformal index, JHEP 08 (2014) 018 [arXiv:1211.6023] [INSPIRE].

[80] I. Gahramanov and V.P. Spiridonov, The star-triangle relation and $3 D$ superconformal indices, JHEP 08 (2015) 040 [arXiv: 1505.00765] [INSPIRE].

[81] A. Gadde and S. Gukov, 2D index and surface operators, JHEP 03 (2014) 080 [arXiv: 1305.0266] [INSPIRE].

[82] F. Benini, R. Eager, K. Hori and Y. Tachikawa, Elliptic genera of two-dimensional $N=2$ gauge theories with rank-one gauge groups, Lett. Math. Phys. 104 (2014) 465 [arXiv: 1305.0533] [INSPIRE].

[83] F. Benini, R. Eager, K. Hori and Y. Tachikawa, Elliptic genera of $2 D \mathcal{N}=2$ gauge theories, Commun. Math. Phys. 333 (2015) 1241 [arXiv:1308.4896] [INSPIRE].

[84] F. Benini and S. Cremonesi, Partition functions of $\mathcal{N}=(2,2)$ gauge theories on $S^{2}$ and vortices, Commun. Math. Phys. 334 (2015) 1483 [arXiv:1206.2356] [INSPIRE].

[85] E. Witten, Phases of $N=2$ theories in two-dimensions, Nucl. Phys. B 403 (1993) 159 [hep-th/9301042] [INSPIRE].

[86] A. Gadde, S. Gukov and P. Putrov, Walls, lines and spectral dualities in 3D gauge theories, JHEP 05 (2014) 047 [arXiv: 1302.0015] [INSPIRE]. 